

\title{
A NURBS Enhanced eXtended Finite Element Approach for Unfitted CAD Analysis
}

\author{
G.Legrain \\ G. Legrain - LUNAM Université, GeM, UMR CNRS 6183, Ecole Centrale de Nantes, 1 rue de la \\ Noë, 44321 Nantes Cédex 3, France \\ Tel.: + 33240372585 \\ fax: +33240372573 \\ Gregory.Legrain@ec-nantes.fr
}

\begin{abstract}
SUMMARY
A NURBS Enhanced eXtended Finite Element Approach is proposed for the unfitted simulation of structures defined by means of CAD parametric surfaces. In contrast to classical X-FEM that uses levelsets to define the geometry of the computational domain, exact CAD description is considered here. Following the ideas developed in the context of the NURBS-Enhanced Finite Element Method, NURBS-Enhanced subelements are defined to take into account the exact geometry of the interface inside an element. In addition, a high-order approximation is considered to allow for large elements compared to the size of the geometrical details (without loss of accuracy). Finally, a geometrically implicit/explicit approach is proposed for efficiency purpose in the context of fracture mechanics. In this paper, only $2 \mathrm{D}$ examples are considered: It is shown that optimal rates of convergence are obtained without the need to consider shape functions defined in the physical space. Moreover, thanks to the flexibility given by the Partition of Unity, it is possible to recover optimal convergence rates in the case of re-entrant corners, cracks and embedded material interfaces.
\end{abstract}

Keywords: X-FEM, NURBS-enhanced FEM, Partition of Unity, CAD

Preprint submitted to: Computational Mechanics

\section{INTRODUCTION}

In recent years and under the impulsion of the work of Prof. Hughes [28, 12], a growing amount of research has been spent on the interaction between CAD (Computed Aided Design) and CAE (Computed Aided Engineering). Indeed, the most popular description format in CAD is B-Rep which is usually based on NURBS parametric surfaces. NURBS (Non-Uniform Rational B-Splines) allow to represent a wide variety of geometrical shapes, and in particular conic sections which are common in CAD designs. On the other side, CAE relies on a large variety of numerical schemes. Finite elements (FEM) is the most widely used method in the solid mechanics community. FEM relies 
on the mesh-based discretization of both unknown field and geometry. Classically, the boundaries of the mesh are polynomial (and usually linear), which means that some geometrical information is lost while discretizing the CAD. Based on this observation, Hughes et al. introduced the so-called isogeometric analysis concept (IGA) where it is proposed to unify the mathematical representation of CAD and CAE. The concept consists in using NURBS (or B-Splines) not-only for the geometrical description, but also for the approximation of the fields involved in the boundary value problem [28]. This allows an exact representation of the geometry in the simulation process. Beside this advantage, NURBS or B-Spline basis allow the construction of arbitrary smooth approximation basis for the treatment of high-order PDEs [24]. IGA has been used in a large variety of studies. For an up-to date overview, the reader can refer to the following special issue [2]. However, IGA still suffers from some partial limitations. The first one comes from the tensor-product nature of the basis functions. This prevents local mesh refinement, which is computationally expensive. To overcome this issue, hierarchical B-Spline (or more recently NURBS) basis can be considered [20, 44, 46]. Alternatively, T-Splines [47] where also proposed as a remedy to this issue. T-Splines allow local refinement thanks to T-junctions and have been used with success in IGA [3]. However, the smoothness of the approximation can be lost, limiting it to $C^{1}$ regularity across T-junctions. A second limitation of NURBS-based IGA concerns the need to decompose the geometry in rectangular or cubic patches (because of the shape of the parametric space). Such a decomposition is not natural for CAD modellers and requires the set of patches to be linked together. Note however that the use of T-spline-based IGA does not constrain the parametric space to be rectangular anymore. The next limitation concerns the treatment of trimmed or singular NURBS that are widely used in real geometries. Recently, a proposition has been made by Kim et al. $[29,30]$ in order to be able to treat complex geometries within only one patch (trimmed by several curves). The last limitation comes from the fact that the effort to implement efficiently IGA in an existing finite element code can be significant. In order to overcome these issues, Sevilla et al. proposed the so-called NURBS-Enhanced Finite Element Method (NEFEM) [49, 50]. This approach allows to consider the exact CAD geometry, yet still using an almost classical FE code. In fact, even finite elements can handle exact geometrical description by means of blending mappings [56]. This class of mapping was introduced by Szabó and Babuška [56] in the context of $p$-Finite Elements which require accurate geometrical description $[56,57]$. In contrast to blending mapping, the NURBS-Enhanced mapping allows the use of trimmed and singular NURBS in both 2D or 3D. NEFEM also introduce the use of cartesian shape functions in order to be able to recover optimal convergence rates that cannot be attained for high-order FEM (due to the definition of the shape functions in the parent space). This method allows to define high-order finite element approximations on exact geometries. Thus, NEFEM can be seen as a good compromise between IGA and FEM (easy implementation, exact geometrical description), although the higher-order continuity of the shape functions and the backward CAD compatibility of IGA are lost. The main drawbacks of this approach concern the treatment of singularities that decrease the convergence rate (note that this is also the case with IGA and $p$-FEM), and sometimes mesh generation that can be complicated due to the creation 
of twisted elements.

In addition to its inability to represent exactly CAD geometry, finite elements can loose their efficiency when dealing with evolving boundaries (such as cracks or material interfaces) or very localized phenomena (such as singularities). Indeed, the computational mesh has to be updated at each step of the evolution in order to be compatible with the geometry (which is also the case with IGA). Partition of Unity finite elements [35] are an answer to these issues: thanks to a proper enrichment of the FE basis, singularities and geometrical or material discontinuities can be taken into account accurately. X-FEM [36] and GFEM [53] are special instance of Partition of Unity FE methods. These methods have been applied to a wide variety of problems. For a recent overview of these methods, the reader can refer to [58, 22]. Partition of Unity methods have been also used as a mean to solve CAD-based unfitted analysis $[6,17,32,39,53]$ i.e. without the burden of meshing. One could also cite fictitious domain approaches such as the Finite-Cell method $[18,41,45,44]$ which has been used with great success in the CAD context. The main limitation of these methods comes from the fact that the geometry is not exactly taken into account. Indeed, it is facetized in the case of the X-FEM because of the interpolation of the Level-Set, and fuzzy in the case of the Finite Cells as only the interior integration points are considered. Only the GFEM which uses blending-mapping and explicit geometry can recover an exact representation (although non-smooth geometries are difficult to take into account in 3D [50]). In addition, note that the X-FEM has already been used in the context of IGA. The first contribution was proposed by De Luycker et al. [15] who applied the X-FEM to IGA on mode I crack problems and studying the influence of BCs and enrichment. It is worth mentioning that in this study, only rectangular physical domains where considered. Ghorashi et al. [23] proposed an eXtended IGA (XIGA) strategy in fracture mechanics, handling crack propagation on NURBS-based domains. In this study, the domain is still rectangular, and the crack is assumed piecewise-linear in both physical and parametric space. Finally, Haasemann et al. [25] proposed a NURBS-based representation of micro-structures. In this study, each sub-element is considered as a NURBS surface which can lead to integration issues in $3 \mathrm{D}$ if breakpoints are located on the interface.

The objective of this work is to propose a NURBS-Enhanced eXtended Finite Element Approach (NE X-FEM in the following). This approach allows to solve unfitted CAD-based BVP, eliminating the meshing burden that can occur both in IGA (when decomposing the geometry into rectangular patches) and NEFEM (where twisted elements can appear). The main ingredients of the approach are (i) the use of NURBS-Enhanced subelements for an exact representation of the geometry; (ii) the use of high-order approximations for a high accuracy with coarse meshes and (iii) the use of enrichments in order to keep the accuracy near re-entrant corners and material interfaces. Finally, the approach allows an implicit-explicit definition of the geometry in order to use the more efficient tool for a given task: NURBS for the geometrical definition of the global domain, and LevelSets for the treatment of evolving details such as cracks or evolving material interfaces. 
The outline of the paper as follows: First, NURBS-Enhanced basics are recalled, and the approach is compared with classical F.E. mapping (isoparametric or blending mapping). In a second section, the NURBS-Enhanced eXtended Finite Element approach is introduced together with implementation details. Next, the approach is validated with simple examples by considering 2D NURBS (or Spline) based geometries in the case of both regular and singular solutions. Then, it is applied to more complex cases with the use of the implicit-explicit approach. Finally conclusions and outlook are given.

\section{NURBS-ENHANCED FEM}

\subsection{NURBS curve / surface}

A $p^{\text {th }}$ order NURBS (Non Uniform Rational B-Spline) $\mathcal{C}$ is a parametric piecewise rational function defined as:

$$
\mathcal{C}(t)=\sum_{i=1}^{n_{c p}} R_{i}^{p}(t) \mathbf{B}_{i}, \quad t \in\left[t_{a}, t_{b}\right]
$$

In this expression, $\mathbf{B}_{i}$ are the coordinates of the $n_{c p}$ control points defining the curve. The piecewise linear interpolation of the control points is called control polygon. The parametric functions $R_{i}^{p}(t)$ are rational functions defined by means of $p^{\text {th }}$ order normalized B-Spline functions $N_{k}^{p}(t)$ as:

$$
R_{i}^{p}(t)=\frac{N_{i}^{p}(t) w_{i}}{\sum_{k=1}^{n_{c p}} N_{k}^{p}(t) w_{k}}
$$

Where $w_{i}$ are the control weights. B-Spline functions $N_{i}^{p}(t)$ are defined recursively following the Cox-de Boor recursion formula $[14,8]$ as:

$$
N_{k}^{0}(t)=\left\{\begin{array}{cc}
1 & \text { if } t \in\left[t_{k}, t_{k+1}[\right. \\
0 & \text { otherwise }
\end{array}\right.
$$

Then,

$$
N_{k}^{p}(t)=\frac{t-t_{k}}{t_{k+p}-t_{k}} N_{k}^{p-1}(t)+\frac{t_{k+p+1}-t}{t_{k+p+1}-t_{k+1}} N_{k+1}^{p-1}(t)
$$

In these expressions, abscissa $t_{k}(k=1 \cdots n+p+1)$ are called knots or breakpoints. These abscissa are assumed to be ordered, so that a so-called knot vector can be formed:

$$
T=\{\underbrace{t_{a}, \cdots, t_{a}}_{p+1 \text { time }}, t_{p+2}, t_{p+3}, \cdots, t_{n-1}, t_{n}, \underbrace{t_{b}, \cdots, t_{b}}_{p+1 \text { time }}\}
$$

The abscissa of the breakpoints may not be distinct: in this case the number of times an abscissa is repeated is called multiplicity of a knot. This feature characterizes the degree of smoothness of the curve at the breakpoints: the curve is $C^{p-1}$ everywhere except at the repeated breakpoints where it is $C^{p-q}$, with $q$ the number of time the 
breakpoint is repeated. See for example Figure 1(a), where the following knot vector was considered for defining a quadratic Spline (which is a special instance of the NURBS, using constant weights $w_{i}=1$ ):

$$
T=\{0,0,0,0.2,0.4,0.6, \mathbf{0 . 8}, \mathbf{0 . 8}, 1,1,1\}
$$

The spline basis in the parametric space associated to this example is depicted in Figure 2: this basis is piecewise polynomial, and it can be seen in Figure 1(a) how does the multiplicity of a knot affects the smoothness of the curve. Note also that usually NURBS surfaces are trimmed, which means that the parametrization is restricted to a subset of the initial parametric space, see Figure 1(b). In general, 2D parametric space can be trimmed by a NURBS curve, see Figure 3 .

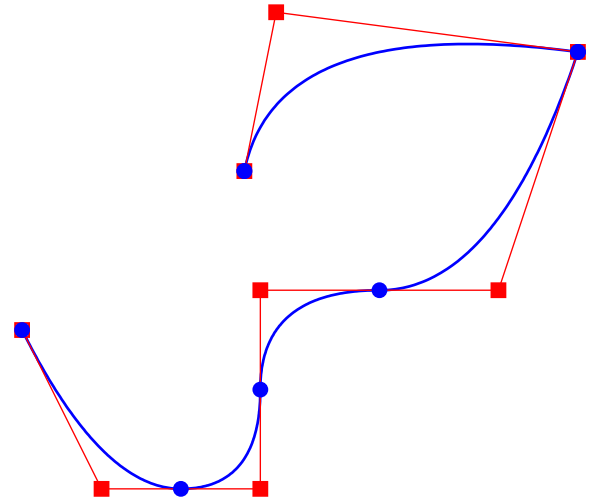

(a)

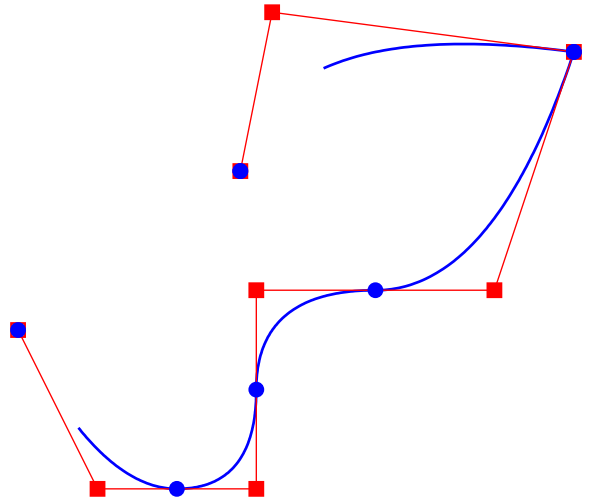

(b)

Figure 1: (a) A piecewise quadratic Spline in 2D (Squares $=$ control points, Dots $=$ image of the breakpoints). (b) The same trimmed Spline.

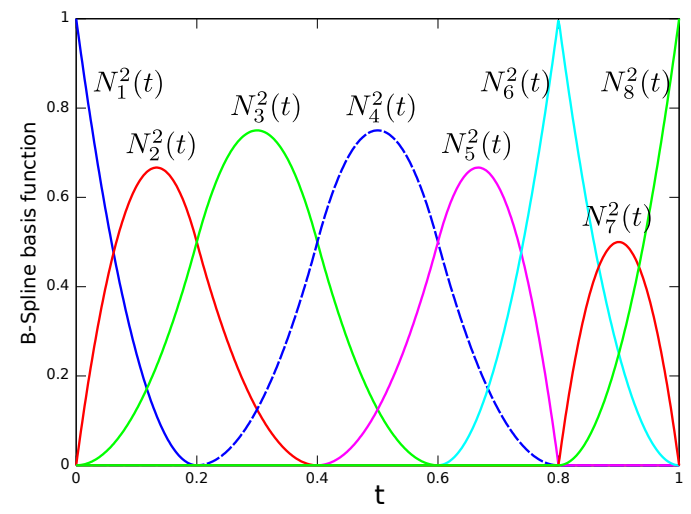

Figure 2: B-Spline basis functions for the knot vector (6). 


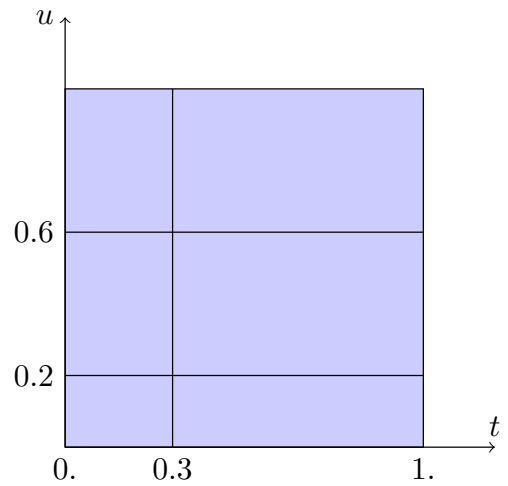

(a)

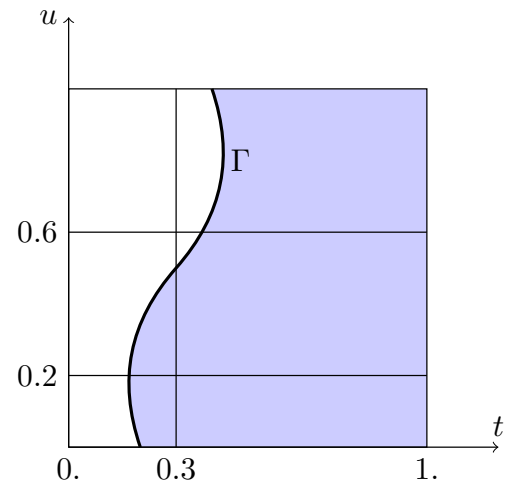

(b)

Figure 3: Trimmed surface. (a) initial parametric space ; (b) parametric space trimmed by NURBS curve $\Gamma$.

In the computational process, the parametric representation of the geometry has to be discretized in order to generate the elements that support the shape functions. However this process can lead to a loss of information, as the rational functions used for the geometrical description cannot be represented by means of conventional mappings. In particular, high-order approximations are known to require a highly accurate boundary representation in order to obtain a proper convergence [57]. Three main possibilities arise in order to ensure geometrical accuracy for conventional FEM: (i) isoparametric mappings [19, 59], (ii) blending function mapping [56] and more recently (iii) NURBSEnhanced finite elements [49, 50] (see [51] for a detailed comparison between these approaches). A second family of methods, based on the isogeometric concept arose recently (coined as isogeometric analysis, (IGA) by Hughes and co-workers [28, 12]). In this context, NURBS are considered, not only for the (exact) geometrical representation, but also for the approximation of the unknown fields. As stated in the introduction, despite its high accuracy, isogeometric analysis can require large modifications of the finite element codes (depending on their architecture) unless the approach proposed by Benson et al. is considered [7]. Moreover, IGA relies on the 3D parametrization of the domain, although usually only the boundary is defined in the CAD. Finally, while being less sensitive to distortions than classical FEM [34], it still needs to partition the mesh in patches in order to ensure good mesh quality. This is why we focus here on the use of an exact geometrical description without any partition of the geometry, while keeping classical extended finite element routines as much as possible.

\subsection{Approximated geometrical representation: Polynomial mapping}

This approach is the most widely used in finite elements when curved boundaries have to be considered. Consider a so called reference element $I$ with local coordinates $\boldsymbol{\xi}=(u, v)$ which is mapped on a physical element $\mathcal{T}_{e}$. In this case, a transformation $\varphi$ is defined 
between the reference element and the physical space, see Figure 4. This transformation is polynomial and in the case of isoparametric finite elements, it is based on the same shape functions as those used for approximating the unknown field:

$$
\mathbf{x}=\boldsymbol{\varphi}(\boldsymbol{\xi})=\sum_{i=1}^{n_{e n}} \mathbf{x}_{i} N_{i}(\boldsymbol{\xi})
$$

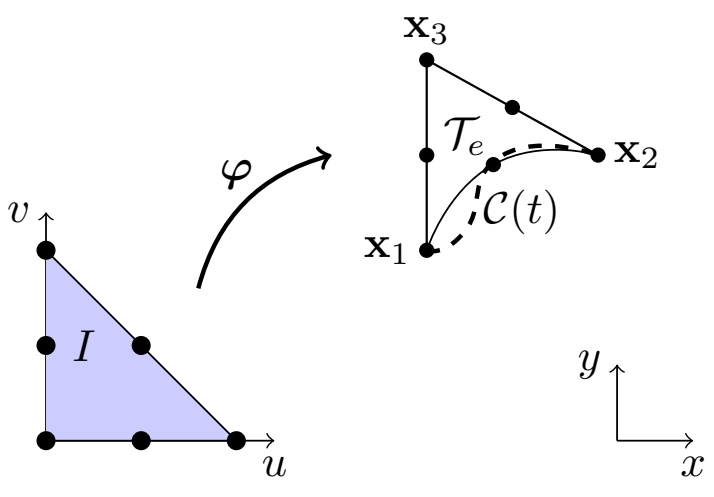

Figure 4: Quadratic polynomial mapping.

This approach is widely used for low order finite elements (linear, quadratic or even cubic) with a regular node distribution. The boundary of the domain is approximated by means of a $p^{\text {th }}$ order polynomial approximation which means that rational functions cannot be represented exactly (see Figure 4). Moreover the bijectivity of the geometrical mapping can be very sensitive to the location of the geometrical nodes for $p \geq 2[11,33]$, which means that in practice finite element codes (and meshers) do not propose geometrical approximations higher than cubic if the nodes are regularly distributed. More recently, Királyfalvi and Szabó proposed a so called Quasi-regional mapping [31]: This approach is based on an improved location of the mapping's collocation points. It is shown that the interpolation error introduced by the polynomial mapping can be mastered, and that nearly $C^{2}$ continuity is obtained between adjacent elements.

\subsection{Exact geometrical representation: Blending Functions}

This mapping was introduced in the context of high-order finite elements ( $p$-FEM) in order to represent exactly the geometry.

For example, if the reference element $I$ depicted in Figure 5(a) has to be mapped on the parametric curve $\mathcal{C}(t)$, the following exact mapping can be defined:

$$
\mathbf{x}=\varphi(\xi)=\frac{1-u-v}{1-u} \mathcal{C}(u)+\frac{u v}{1-v} \mathbf{x}_{2}+v \mathbf{x}_{3}
$$


This mapping has to be used with care in the case where breakpoints are located on the edges of the physical element (which will occur in the NE X-FEM): Because of the loss of smoothness of the geometrical shape functions across these points, accurate integration is obtained only if the reference element is partitioned according to the location of these breakpoints (see Figure 5(b)). This process makes the integration exact but can become cumbersome, especially in 3D (see Sevilla et al. [51]). In the case of $p$-FEM, multiple elements are used along $\mathcal{C}(u)$, so that no breakpoint is located in the reference element.

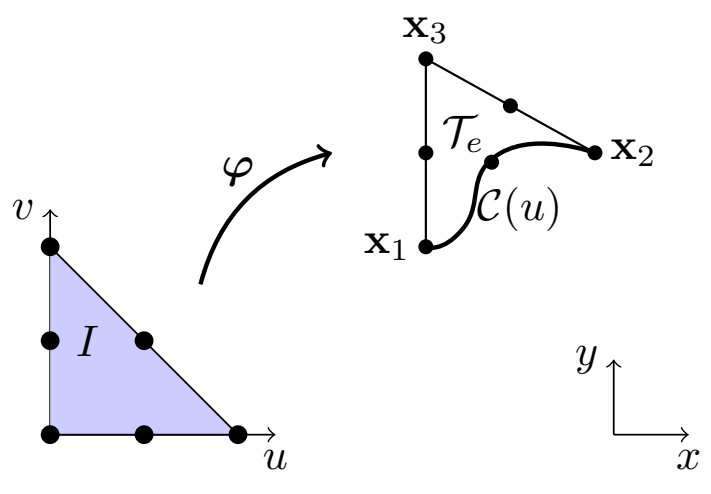

(a)

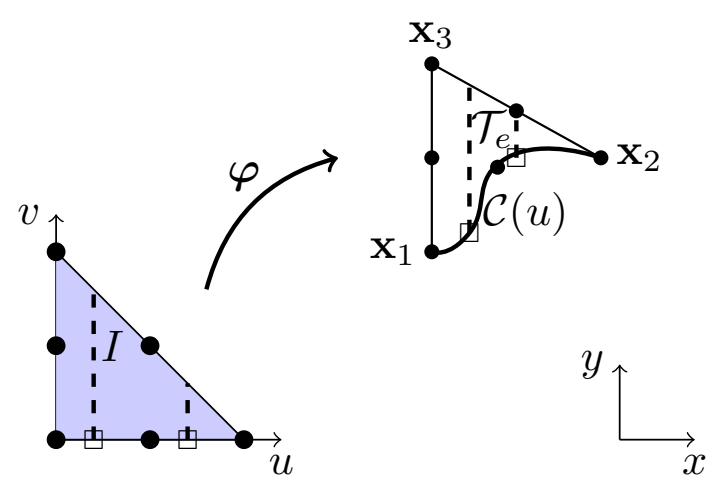

(b)

Figure 5: (a) Exact blending mapping; (b) Composite numerical quadrature with breakpoints (represented as $\square$ )

\subsection{Exact geometrical representation: NURBS Enhanced Mapping}

In order to simplify the definition of the mapping between the reference and physical spaces, Sevilla et al. [49] introduced the so-called NURBS-Enhanced Finite Element Method (NEFEM). The main features of this approach are: (i) The use of a highorder approximation by means of cartesian shape functions and (ii) The definition of a dedicated mapping to integrate the weak formulation of the problem on NURBS-based geometries.

Remark: The use of cartesian shape functions allows to recover the consistency of the approximation for $p$-FEM and isoparametric FEM because the shape functions are not polynomial anymore in the physical space (as $N_{i}(\mathbf{x})=N_{i}(\varphi(\xi))$ ). In the following, the geometrical mapping associated to the approximation mesh will remain linear which means that the polynomial space stay polynomial in the physical space.

The proposed mapping allows to represent exactly the geometry and can be extended to trimmed surfaces (which is not the case for blending mapping and IGA). Consider the physical element depicted in Figure 6(a): The NURBS-enhanced mapping that allows a proper integration of the weak formulation on the physical element is written as the tensorisation of the parametric space restricted to the current element (i.e. $\left.\left[u_{1}^{e}, u_{2}^{e}\right]\right)$ and $[0,1]$ : 


$$
\begin{gathered}
(u, \theta) \in R=\left[u_{1}^{e}, u_{2}^{e}\right] \times[0,1] \\
\boldsymbol{\psi}(u, \theta)=(1-\theta) \mathcal{C}(u)+\theta \mathbf{x}_{3}
\end{gathered}
$$

Note that the proper numbering of the nodes which is necessary for the mapping is easily obtained. The main features of this mapping are the following:

- It exhibits a clear separation between the NURBS direction and the interior direction, which means that one gets an additional flexibility for choosing proper integration rules (the influence of the NURBS is restricted to $u$, whereas it is spread over the whole reference element in the case of the use of a blending mapping).

- The geometrical mapping $\boldsymbol{\psi}$ is linear with respect to $\theta$, therefore the integrals in this direction can be computed exactly for $p^{\text {th }}$ order elements using a 1D GaussLegendre integration rule with $p+1$ points.

- If breakpoints $u_{1}^{b}$ and $u_{2}^{b}$ are located in $\left[u_{1}^{e}, u_{2}^{e}\right]$ (see Figure 6(b)), composite 1D Gauss-Legendre integration rules are considered along $u$ : $\left[u_{1}^{e}, u_{1}^{b}\right],\left[u_{1}^{b}, u_{2}^{b}\right],\left[u_{2}^{b}, u_{2}^{e}\right]$.

- In the case of Spline surfaces, the integration of the weak formulation can be exact, whereas it is approximated for NURBS surfaces (this is also the case with isogeometric analysis and blending mappings). In this case the number of integration points is increased, following [48].

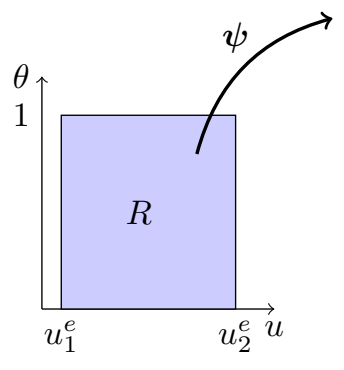

(a)
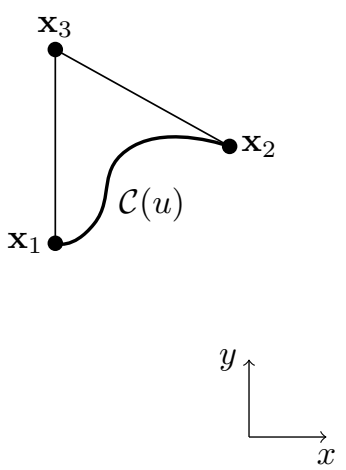

$x$

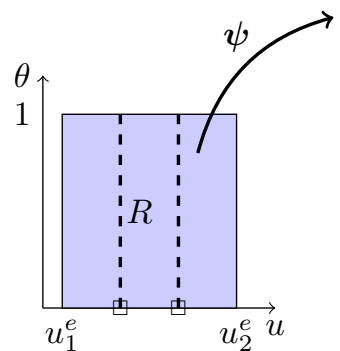

(b)
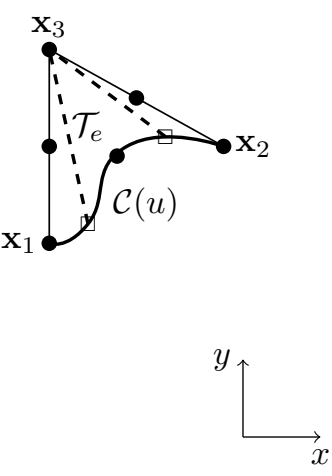

Figure 6: (a) NURBS Enhanced mapping; (b) Numerical quadrature with breakpoints (represented as $\square$ )

\section{NURBS-ENHANCED X-FEM}

The objective of this contribution is to propose an efficient strategy for the non-conforming analysis of CAD structures, taking into account exact geometries (see Figure 7). The 
method is based ${ }^{1}$ on a high-order approximation that allows to obtain highly accurate models even with coarse meshes. The objective is also to answer to some of the issues raised by NEFEM and previous attempts to couple IGA and X-FEM. For instance, the use of straight-sided sub-parametric elements allows to define a consistent high order polynomial approximation as the Jacobian matrix linking reference and physical spaces is constant (for simplices). This avoids the computation of the Vandermonde matrix which is used to define cartesian shape functions on the elements. Moreover, the knowledge of the exact CAD description allows to predict singularities in the solution and use the partition of unity to enrich it and improve the convergence properties. The method is based on the definition of NURBS-enhanced subelements in order to keep the exact representation of the geometry. As in the case of NEFEM, the additional work needed to take into account the exact boundaries is restricted to a small number of elements (those that are crossed by the boundary). No special treatment is needed for the other elements, which differs with isogeometric analysis. Moreover $h$-refinement is easy to implement (even in the anisotropic case), which is not the case for isogeometric analysis due to its tensor product structure. We propose also an implicit-explicit approach for the treatment of evolving details. For instance, cracks are features that naturally evolve in the physical space with unknown smoothness. To the opinion of the author, trying to make it live in the parametric space by pulling-back the physical geometrical informations introduce numerous difficulties (in particular for the consistent update of the crack shape and the integration of the weak form).

With respect to the classical X-FEM approach which uses level sets, the proposed approach allows to take into account the exact geometry and not an approximation (even in the case of high-order level sets $[9,27])$. Similar ideas were also already considered by Strouboulis et al. [54] in the context of the GFEM method and Haasemann et al. [25] for the X-FEM. In contrast, (i) the focus is put here on the use of very high order approximation, (ii) an implicit/explicit definition of the geometry is presented and (iii) the proposed approach is more efficient and easier to implement in 3D thanks to the flexibility of the NURBS-Enhanced mapping.

\subsection{NURBS-Enhanced subelements}

Consider a NURBS boundary which crosses a computational element (see Figure 8). The objective is to take exactly into account the geometry of the NURBS interface for a non-conforming analysis. The approach consists in defining NURBS-enhanced integration subelements for the proper evaluation of the weak form. The integration rule is based on the NURBS-enhanced mapping presented in the previous section. The process involves three steps (see Figure 9):

1. Detection of the elements cut by the interface. This step involves the research of intersections between the edges of the current element and the NURBS. Note that no distance has to be computed, which improves the robustness as the non-linear distance problem can fail to converge depending on its initialization;

\footnotetext{
${ }^{1}$ Not necessarily
} 


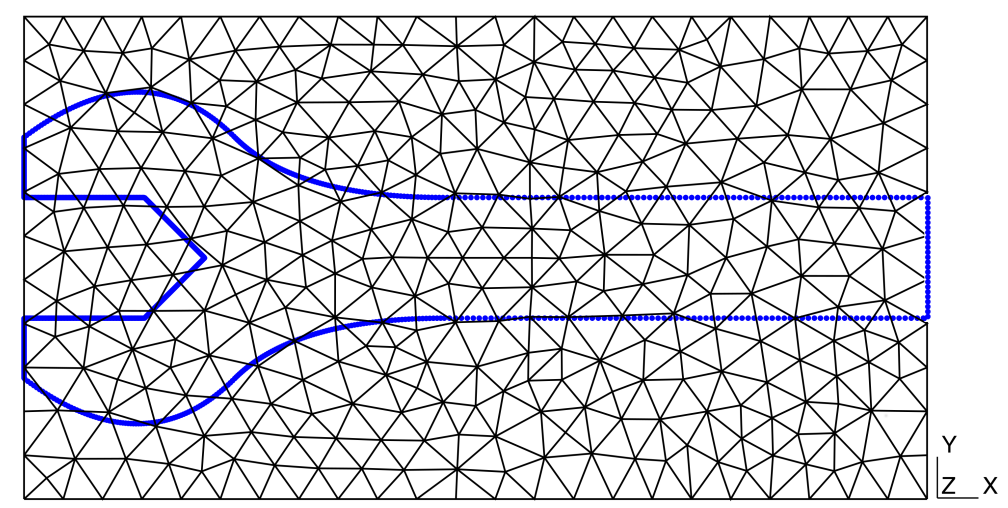

Figure 7: Non-conforming isogeometric analysis of a spanner.

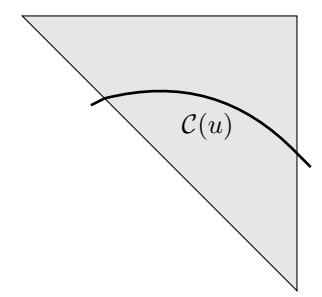

Figure 8: Element cut by a NURBS interface.

2. Construction of the intersections ( $\bullet$ in Figure 9.2). The intersections between the NURBS and the edges of the element are computed, and the parameter range of the curve inside the element is obtained;

3. Construction of the straight subelements based on the intersection points (Figure 9.3). At this point, the ordering of the nodes of the NURBS-enhanced subelements is prescribed, so that it follows the one considered in the definition of the mapping (see equation (9) and Figure 6(a)). In this case, two among the three created subelements will use the NURBS-enhanced mapping (red and blue triangles in Figure 9.3)

4. Finally, the NURBS-enhanced mapping is applied to the curved subelements in order to construct a proper integration rule (Figure 9.4).

During the process, the validity of the NURBS-enhanced subelements is checked. In particular, the elements must have no self-intersection (see Figure 10(a)). In this case, the diagonal of the quadrilateral subelement is swapped as in Figure 10(b). In some cases, the curvature of the NURBS boundary may be too high, so that swapping the diagonal cannot produce any valid subelement (see Figure 11). In this case, the two problematic subelements are recursively split until valid subelements can be created 


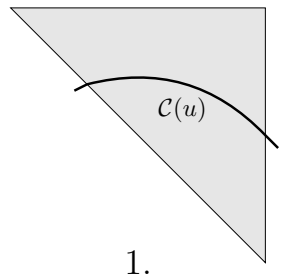

1.

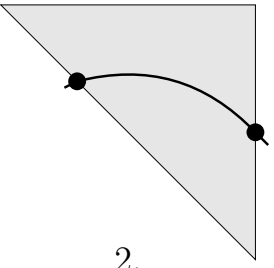

2 .

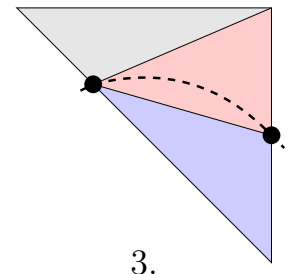

3.

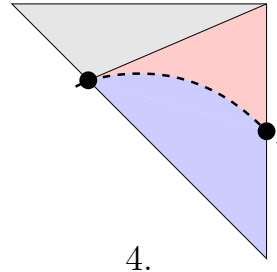

4.

Figure 9: Process for constructing NURBS-enhanced subelements.

(see Figure 11). The resulting computational setup is thus composed of a finite element approximation defined on a background grid of high-order sub-parametric finite elements (linear geometrical mapping). The completeness of the approximation is thus ensured. On the other hand, the geometry (i.e. the subelements) is represented by means of rational functions, but only for the elements that are intersected by the interface.

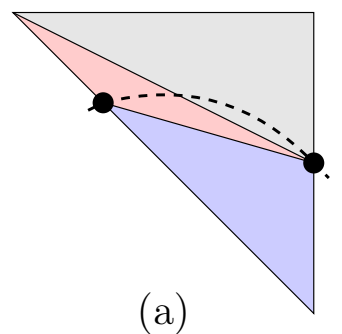

(a)

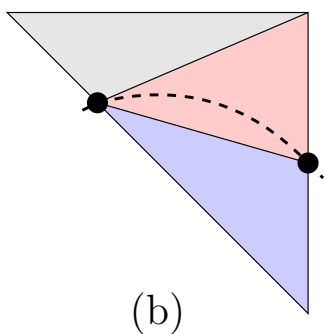

(b)

Figure 10: Checking NURBS-enhanced subelements validity: (a) Red subelement is invalid; (b) Swapping the diagonal to ensure validity.

Once the subelements have been created, proper integration rules can be defined for the integration of the weak form. Classical subelements use Cowper [13] integration rule, whereas NURBS-enhanced subelements use the $1 \mathrm{D} \times 1 \mathrm{D}$ tensorized integration rule presented in section 2.4 .

\subsection{Illustration}

The approach is now illustrated on a simple example depicted in Figure 12(a) consisting of a $2 \mathrm{D}$ domain containing a circular hole at its center. The free surface of the hole is represented my means of the union of four quadratic (order 3) NURBS that allows an exact representation of the shape. The domain is meshed by means of straight triangles that are independent of the hole (see Figure 12(b)). Then, NURBS-enhanced subelements are created, following the methodology presented in the previous section (see Figure 12(c)). Finally, the NURBS-enhanced integration rule is applied on those elements. Figure 12(d) and (e) show the location of these integration points in the physical space. 


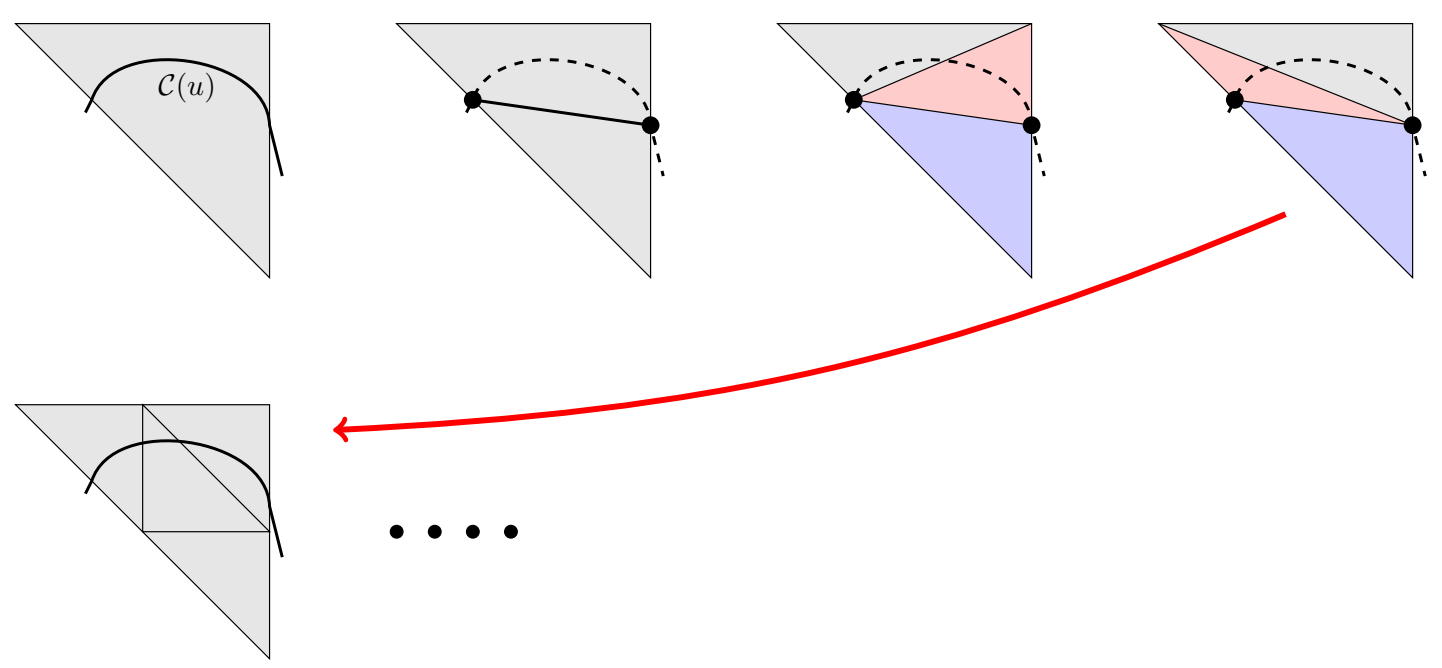

Figure 11: Recursive subdivision of the subelements to ensure the validity of the NURBSenhanced subelements.

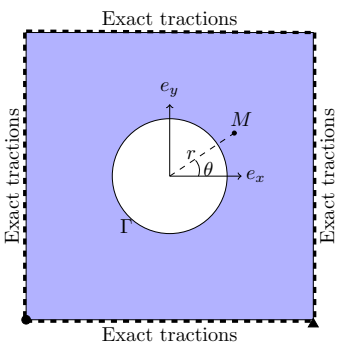

(a)

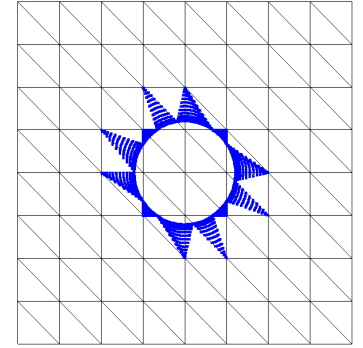

(d)

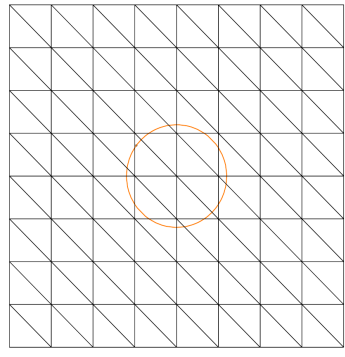

(b)

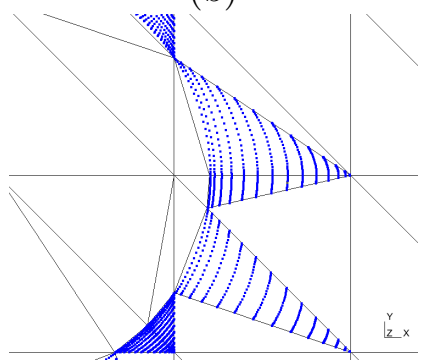

(e)

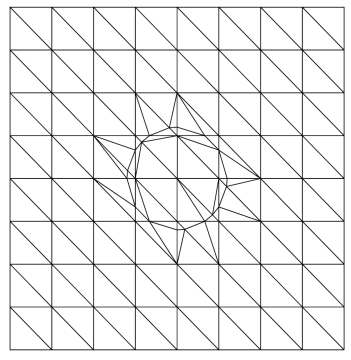

(c)

Figure 12: Square 2D domain containing a circular hole. (a) Geometry; (b) Mesh and hole NURBS; (c) Mesh and subelements; (d) Integration points in the domain ; (e) Zoom. 


\section{VERIFICATIONS}

The method is now applied on structures containing boundaries defined by means of NURBS and Splines. Focus is put on the convergence properties of the approximation in the case of both regular and singular solutions. All the problems assume a linear elastic material behaviour and the small strain assumption.

\subsection{Plate with a hole}

Consider an infinite plate with a circular hole in its center in uniaxial tension $\sigma_{\infty}=$ 1. MPa along $\mathbf{x}$ axis (see Figure 12(a)). The analytical solution of this problem is given in [55] and is used here as a reference solution for error monitoring. Only one fourth of the problem is considered: a square of length $L=2 \mathrm{~mm}$ with a circular hole of radius $a=1.0 \mathrm{~mm}$ at its bottom left corned is used here. Finally, tractions computed from the exact solution are applied on the boundary of the domain with symmetry conditions along the left and bottom edges. The exact solution of this finite problem is therefore the same as the exact solution inside the boundary. Young's modulus is set to $1.0 \mathrm{MPa}$ and Poisson's ratio to 0.3. Typical mesh and integration points location are depicted in Figure 12(d) and (e). First, the surface error is evaluated for the numerical model by the integration of a unit function over the domain. In practice, this error is near machine precision, which shows the geometrically exact nature of the proposed strategy. Then, a $h$-convergence study is performed by considering meshes from 2 to 32 elements per side and polynomial order from 1 to 6 (using Bernstein shape functions). The convergence curves are presented in Figure 13: it can be seen that optimal convergence rates are obtained from linear to hexic polynomial approximation. The slightly lower rate of convergence in this latter case is due to a lack of accuracy in the integration of the exact solution during error computation (typical error level on the domain is between $10^{-15}$ and $10^{-11}$ ). The raw stress field obtained from the hexic computation on a $9 \times 9 \times 2$ triangular mesh is presented in Figure 14. Finally, a $p$ convergence is conducted on a $2 \times 2 \times 2$ triangular mesh (see Figure 15): as the solution is analytic, exponential convergence is obtained. 


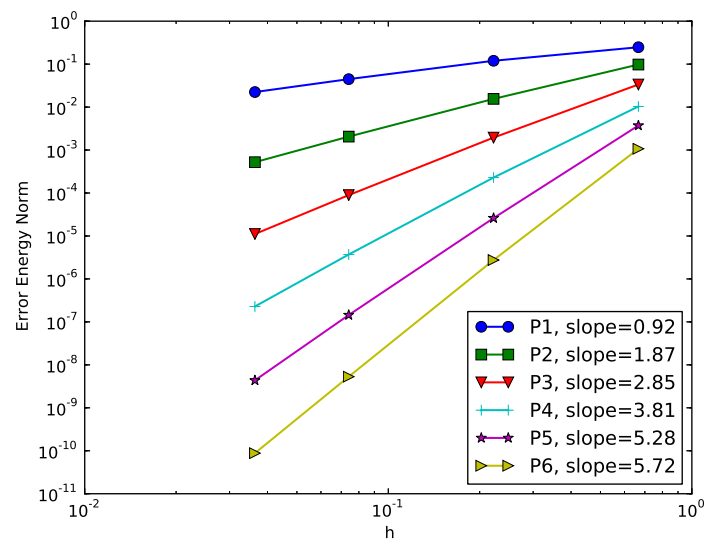

Figure 13: Infinite plate containing a hole: $h$-convergence.

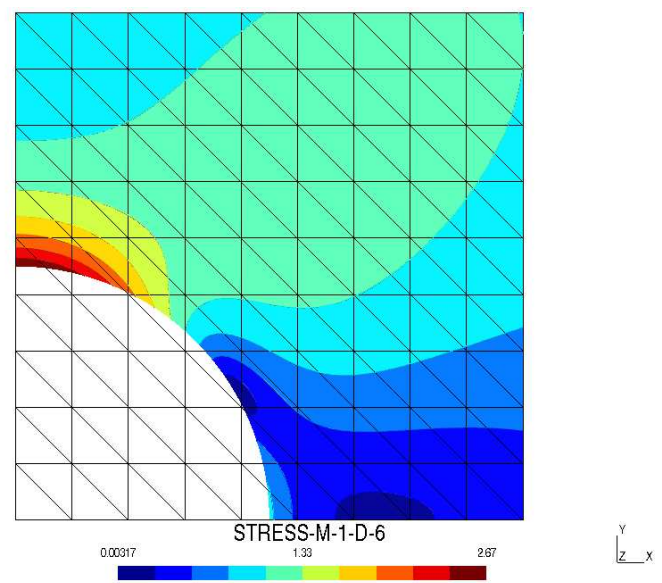

Figure 14: Infinite plate containing a hole: Raw stress state for a $9 \times 9 \times 2$ triangular mesh using a $P 6$ interpolation. 


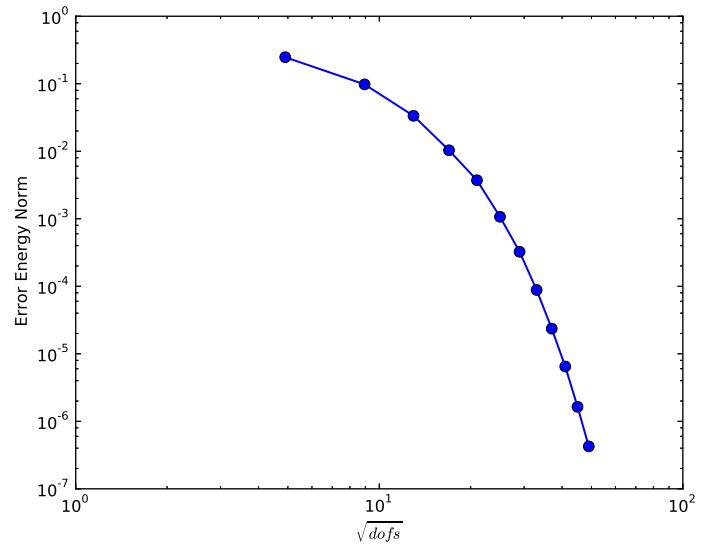

Figure 15: Infinite plate containing a hole: $p$-convergence. 


\subsection{L-Shaped Panel}

The next example focus on the improvement of the convergence behaviour by means of suitable enrichment functions. Consider the L-Shaped panel depicted in Figure 16. A Laplacian equation has to be solved on this domain, with the boundary conditions depicted in Figure 16. The exact solution $u(r, \theta)$ of this problem is singular :

$$
u^{e x}(r, \theta)=r^{2 / 3} \sin \left(\frac{2 \theta}{3}+\frac{5 \pi}{6}\right)
$$

Because of the singular solution, the convergence rate of the finite element method is bounded by the order of the singularity (i.e. $2 / 3$ here, see [56]) unless the solution is enriched. This bound on the convergence rate is particularly harmful with highorder approximations. Classically, graded $h-p$ meshes are used in order to overcome this issue, but this is somehow contradictory with the philosophy of the NEFEM (i.e. small details do not implies small elements). Otherwise, the use of the eXtended Finite Element Method allows to enrich the solution with singular terms ( $u^{e x}$ in this case) and recover the rates of convergence with a smooth solution. Here, the corner of the panel is represented by means of the linear Spline presented in Figure 17 on top of a typical unstructured mesh used for the convergence study. This study is performed on a sequence of successively refined meshes. Only linear, quadratic and cubic approximations are considered here, both enriched or not. In the enriched case, a circular zone of radius 0.3 is considered for selecting the enriched dofs. The integration strategy consists in the use of a larger number of integration points in the enriched zone. Note that here a classical Gauss quadrature was considered: more adapted integration rules could also be used. Typical integration points are depicted in Figure 18. Note that the red edge in these two figures is mapped onto two orthogonal segments.

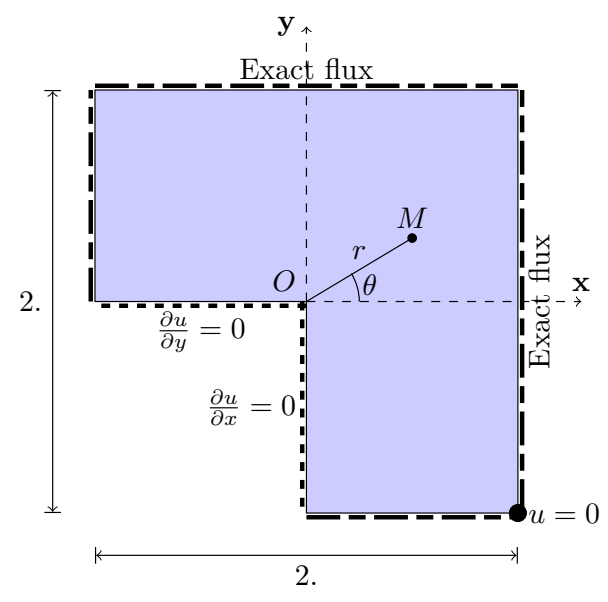

Figure 16: L-Shaped Panel: Domain and boundary conditions 


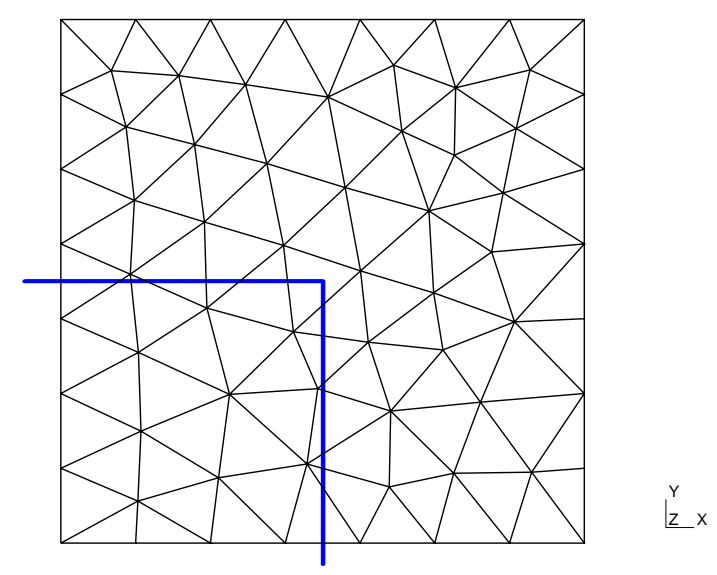

Figure 17: L-Shaped Panel: Spline defining the corner and typical mesh.

The results of the convergence study are presented in Figure 19: As expected, convergence rates for non-enriched approximations are bounded by the order of the singularity. In contrast, nearly optimal convergence rates can be recovered due to the enrichment. Optimal convergence rates are difficult to obtain here because of the integration of singular terms due to the enrichment function. Here, very high-order Gauss quadratures had to be used in the enriched area $(63 \times 63$ points in the $u \times \theta$ space $)$.

In a second step, the flexibility of the approach is illustrated by studying variations of the design of the plate. A variable fillet is now added in the re-entrant corner of the plate. The radius of this fillet is set to $0.01,0.05$ and 0.1 . The boundary conditions are modified according to Figure 20, and a P6 approximation is considered. The norm of the flux for different radii are depicted in Figure 21. It is shown that changes in the design could be tested without any modification in the mesh (which should be rebuilt and repartitioned into blocks with IGA when the filled is introduced and when the radius of the fillet is modified). 


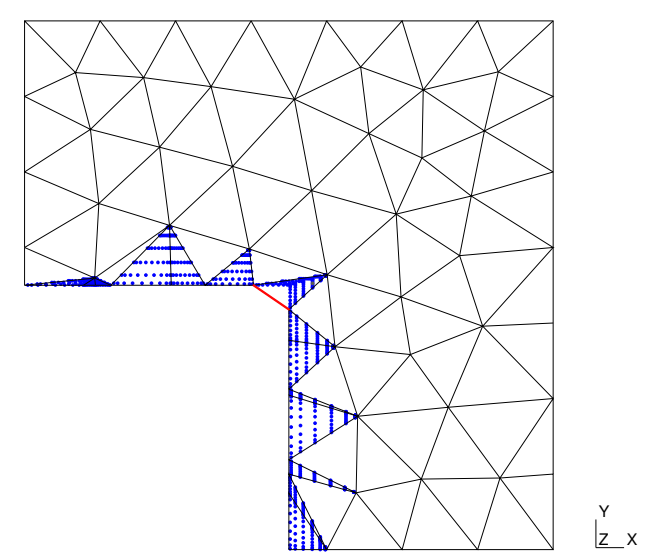

(a)

(b)

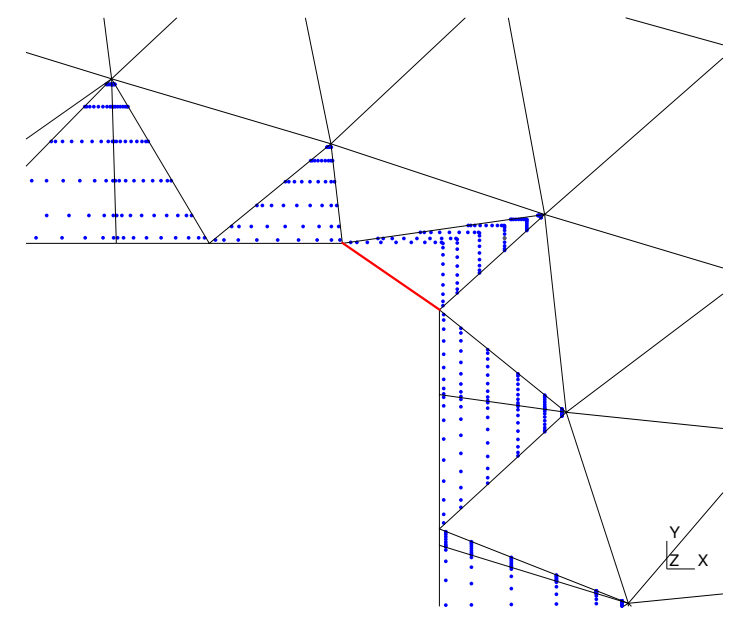

Figure 18: L-Shaped Panel: Integration points ((a) Overview; (b) Zoom). Note that the red edge is mapped onto the corner. 


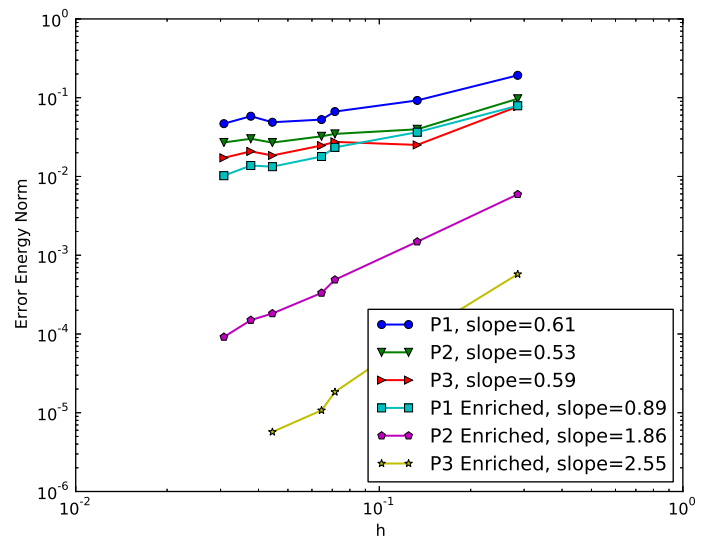

Figure 19: L-Shaped Panel: $h$ convergence

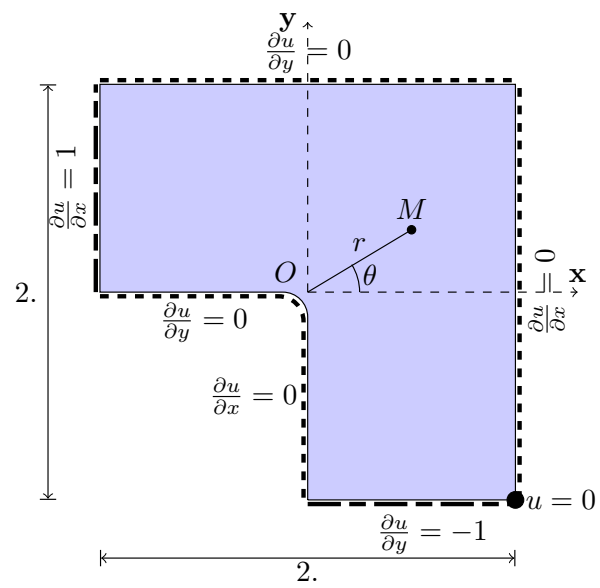

Figure 20: L-Shaped Panel with a fillet: Domain and boundary conditions 

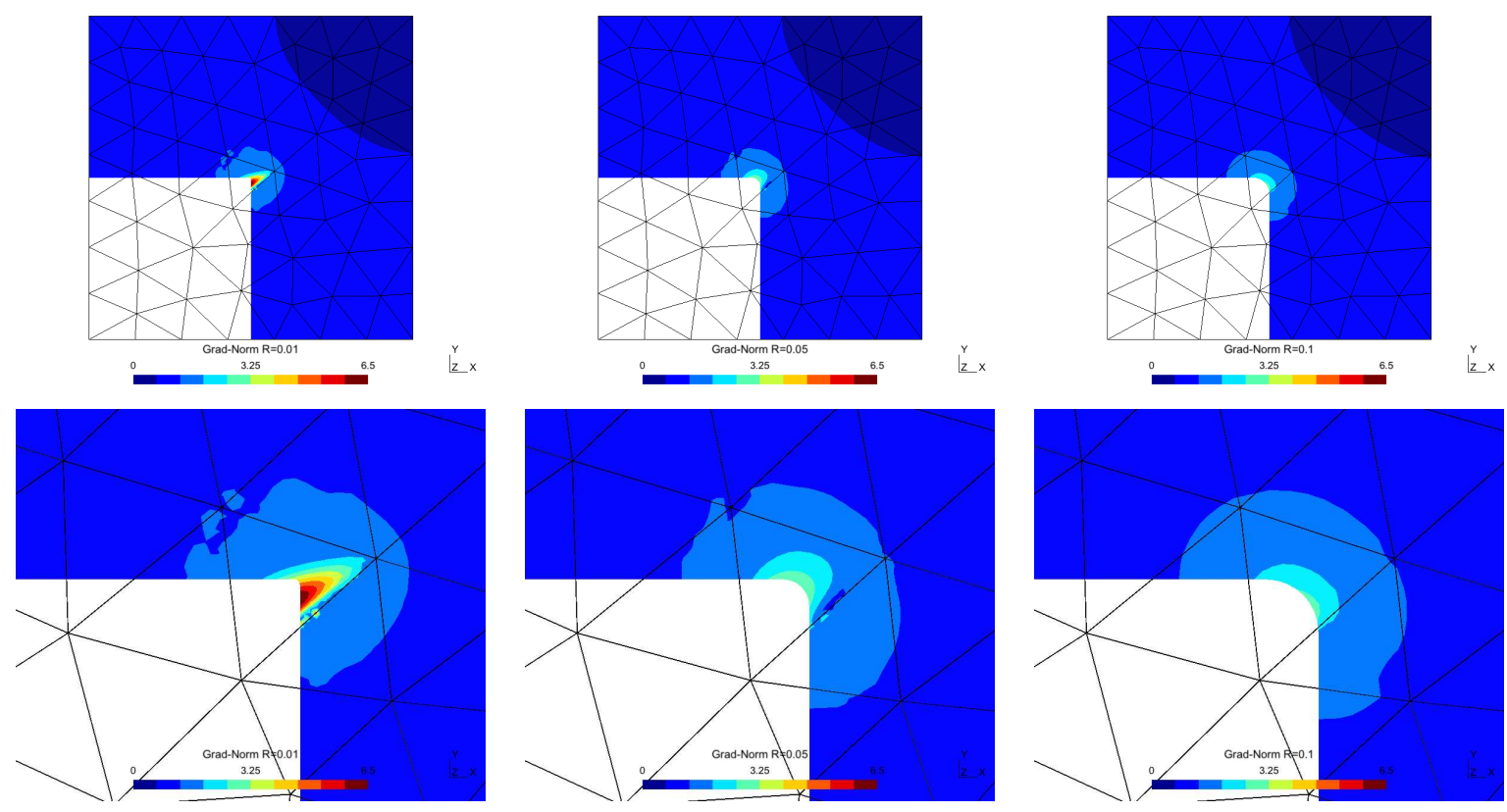

Figure 21: L-Shaped Panel flux: Influence of a fillet at the corner of the panel. 


\subsection{Material interfaces}

The approach is now applied to a non-conforming material interface: indeed, the example studied in the last section could have been treated with NEFEM or IGA with the use of the asymptotic field near the corner as an enrichment. This is not the case with the present example: Consider an infinite plate subjected to equibiaxial tension and containing a circular inclusion of radius $a=1 . \mathrm{mm}$ at its center. The approximation domain consists in one fourth of the model, i.e. a square of length $2.0 \mathrm{~mm}$ centred on point $(1,1)$ (see figure 22). The two materials are considered as linear elastic with Young's modulus $E_{m}=1.0 \mathrm{MPa}$ and Poisson's ratio $\nu_{m}=0.3$ for the matrix. Concerning the inclusion, the following parameters are considered: $E_{i}=10.0 \mathrm{MPa}$ and $\nu_{i}=0.25$. The analytical solution for this problem was given in [55] and is used to apply exact tractions on the boundary of the approximation domain. The material interface is represented using the proposed approach, and the approximation is enriched by means of the so called 'abs' enrichment function [55], which represents the distance of a given point to the interface. In has been shown that such an enrichment function induces blending elements that degrades the convergence rate of the approximation [55, 37, 10, 21]. Various enrichment functions have been proposed in order to recover an optimal convergence rate for both low and high order approximations [37, 21, 9, 17, 1, 32, 43]. In this contribution, the so-called corrected X-FEM is considered [21]. First a $h$-convergence is considered, using regular finite element meshes: The error in the energy norm is plotted with respect to mesh size in Figure 23(a). It can be seen that optimal (or close to optimal) convergence rates are obtained from $\mathrm{P} 1$ to $\mathrm{P} 4$ approximations. Finally, a $p$-convergence is considered, using a regular $3 \times 3 \times 2$ triangular mesh (the last $\times 2$ comes from the fact that the mesh is obtained by splitting a $3 \times 3$ quad mesh into two triangles). The approximation ranges from $\mathrm{P} 1$ to $\mathrm{P} 7$ and the results are plotted in Figure 23(b). It can be seen that an exponential convergence is obtained as in [32].

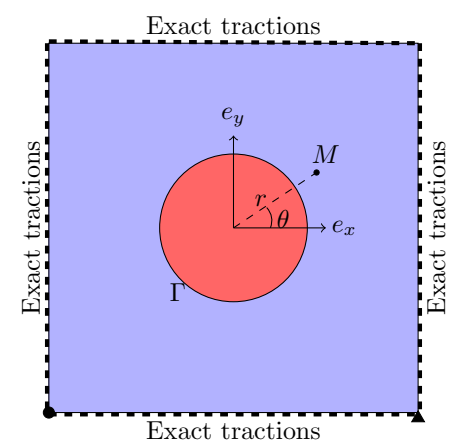

Figure 22: Material interface: computational domain and boundary conditions. 
(a)

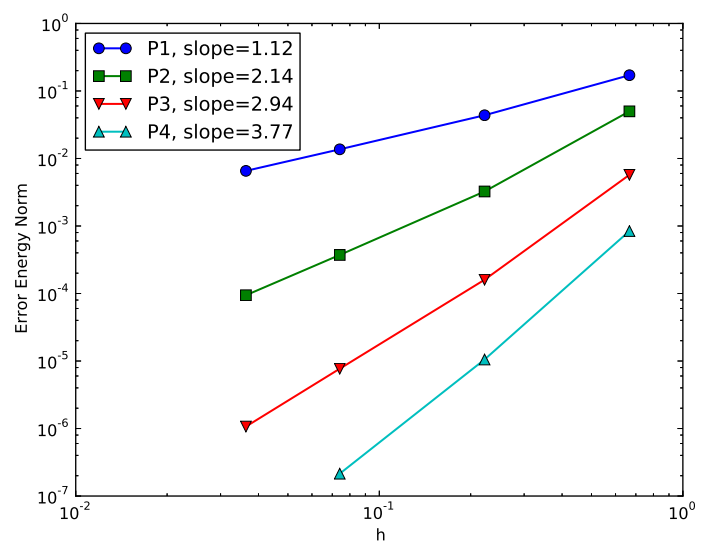

(b)

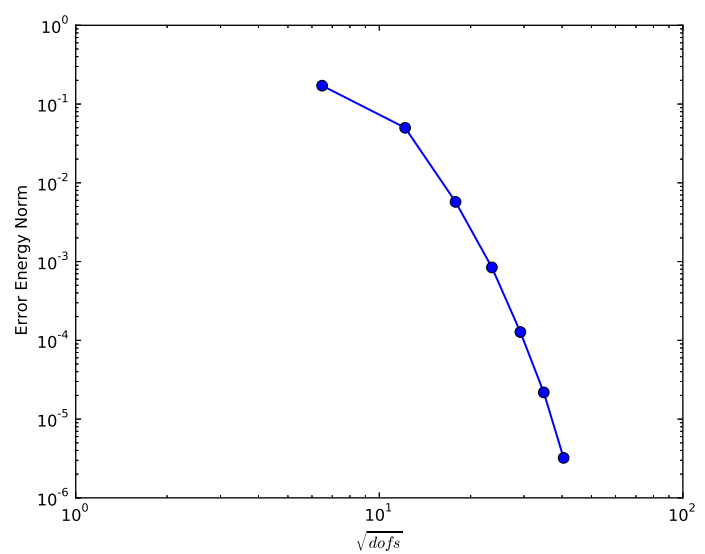

Figure 23: Material interface: (a) $h$-convergence; (b) $p$-convergence.

\subsection{Spanner}

As a more complex example, consider the simulation of a spanner-like structure defined by means of a third order Spline. The geometry of the spanner and the computational mesh were already presented in Figure 7. The spanner is assumed to be made from a linear elastic material with unit Young's modulus and Poisson's ratio equal to 0.3. The boundary conditions are depicted in Figure 24. The Dirichlet BC near the head are enforced by means of a penalty approach with parameter equal to $10^{6}$ (note that Lagrange multipliers $[38,5]$ or Nitsche's method $[40,26,16]$ could be equally considered). The problem is solved using a hexic polynomial interpolation and the mesh depicted in Figure 25, which leads to 9410 dofs. This solution is compared to a quadratic finite element computation, using the mesh depicted in Figure 26(a). Finally, a reference solution is computed using quadratic finite elements and the mesh depicted in Figure 26(b). These finite element solution involve respectively 10593 and 200421 dofs, and their correspond- 
ing Von-Mises stress fields on the deformed configuration are depicted in Figure 27(a-b).

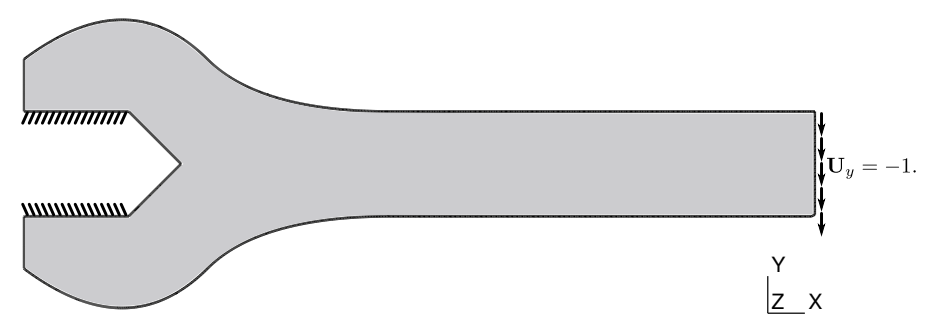

Figure 24: Spanner: Geometry and boundary conditions.

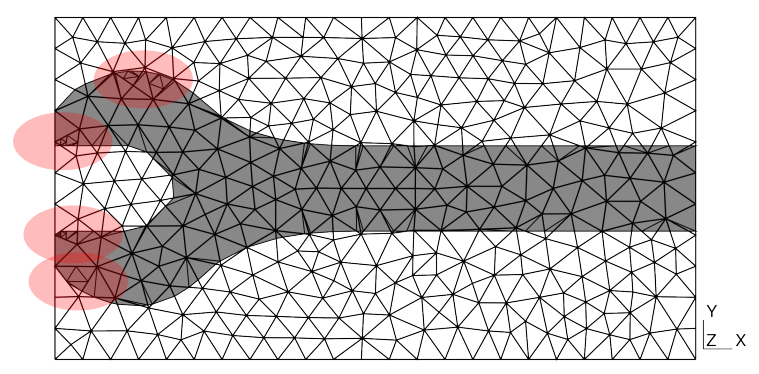

Figure 25: Spanner: X-FEM matter zone and recursive subelements.

The matter part of the X-FEM domain is depicted in Figure 25. Subelements that had to be split according to Figure 11 are highlighted. The solution (Von-Mises stress field on the deformed configuration) is presented in Figure 28. It can be seen that this solution compares favourably with the finite element ones (Figure 27(a-b)). More precisely, the discrepancy in the strain energy between NE X-FEM and FEM solutions are presented in table 1. Although the error level of the NE X-FEM computation is small, the approach is less efficient in term of dofs than the conforming solution. This stems from two reasons: (i) The solution involves three singular points near the head of the spanner. The coarse high order mesh is not able to capture such a solution as the convergence is only algebraic in this case. The enrichment of the approximation would solve this issue. (ii) Numerical tests have highlighted a dependency on the penalty parameter for such polynomial order. This is why a second mesh is proposed in order to make the influence of the BCs vanish. This mesh is depicted in Figure 29(a) and is said boundary fitted as the dirichlet boundary condition is applied exactly. The resulting energy error is given in table 1: the approach is now more efficient than the conforming one. However, the singularities are still not properly represented (see Figure 30(a)). In order to capture these features, two possibilities are considered: first, a graded mesh is considered in Figure 29(b). This mesh is extremely coarse in the areas where the fields are smooth and refined near the singular points. The results are given for a P5 approximation as it leads to a similar number of dofs as the other computations (see 
(a)

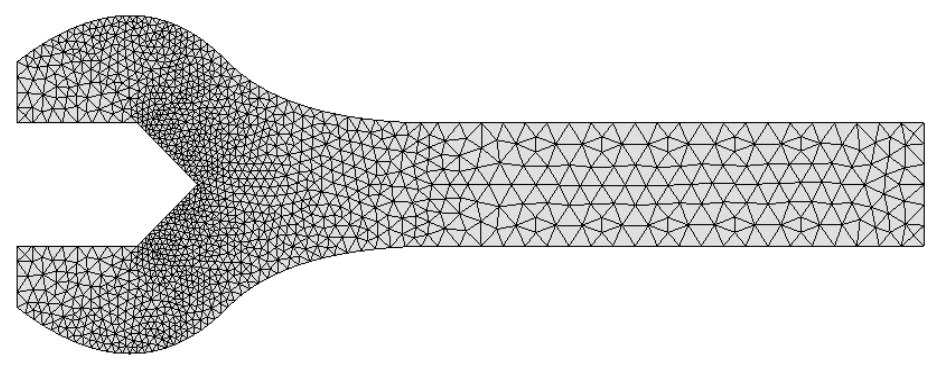

(b)

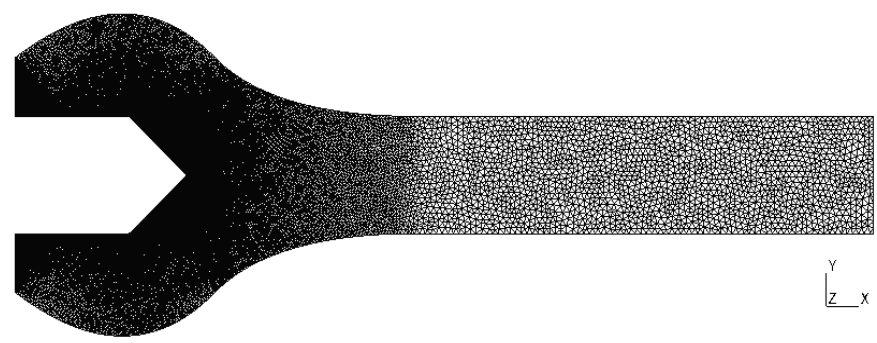

Figure 26: Spanner: (a) FEM mesh (2570 triangles); (b) Reference FEM mesh (49 781 triangles)

\begin{tabular}{ccc} 
Computation & Err / Ref & Dofs /Ref \\
\hline \hline FEM quadratic & $0.39657 \%$ & $5.3 \%$ \\
NE X-FEM (P6) & $0.61391 \%$ & $4.7 \%$ \\
NE X-FEM Bnd fitted (P6) & $0.33992 \%$ & $5.17 \%$ \\
NE X-FEM Bnd fitted graded (P5) & $0.11239 \%$ & $4.7 \%$ \\
NE X-FEM Bnd fitted enriched (P5) & $0.10222 \%$ & $4.8 \%$ \\
\hline \hline
\end{tabular}

Table 1: Spanner: Errors with respect to the reference solution

table 1 and Figure 30(b)). It can be seen that the improvement is spectacular (the error level is divided by 3). Second, the mesh is kept homogeneous (see figure 29(a)) and the approximation is enriched near the singular points by means of adapted enrichment functions (see [52] for the derivation of asymptotic fields near corners). The enrichment is geometric using circles of radius $0.5 \mathrm{~mm}$ from the singular points. A P5 approximation is still considered and it can be seen in table 1 that the accuracy is similar to the one obtained using the graded mesh. This validates the proposed approach and highlights the importance of the proper enforcement of the boundary conditions. 
(a)

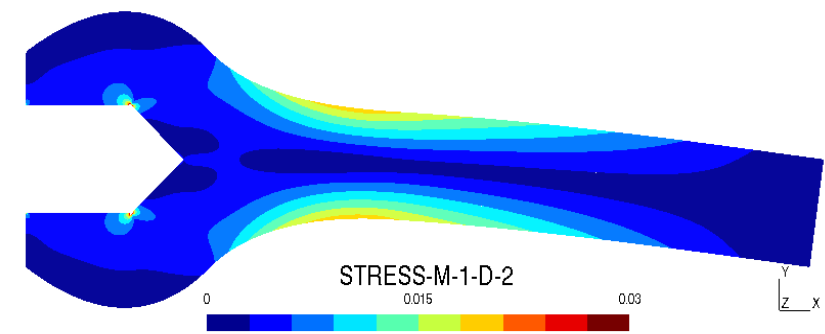

(b)

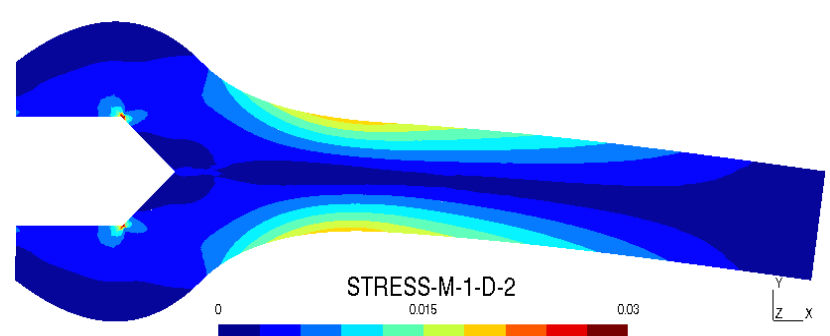

Figure 27: Spanner: FEM solutions, quadratic approximations: (a) Reference solution (using mesh 26(a)) ; (b) Using mesh 26(b) (Von-Mises norm of the stress field on the deformed configuration).

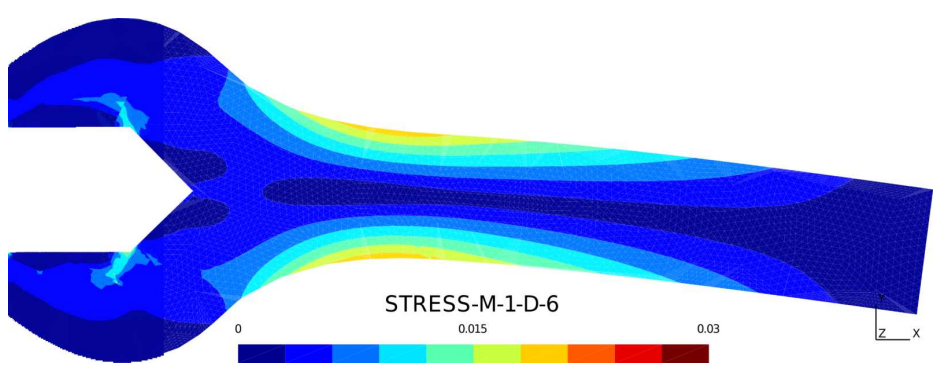

Figure 28: Spanner: X-FEM solution (Von-Mises norm of the stress field on the deformed configuration). 
(a)

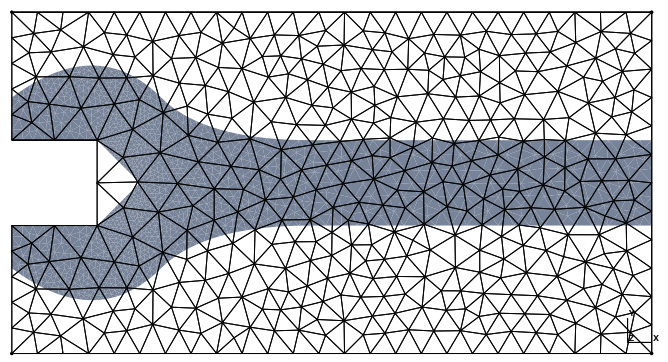

(b)

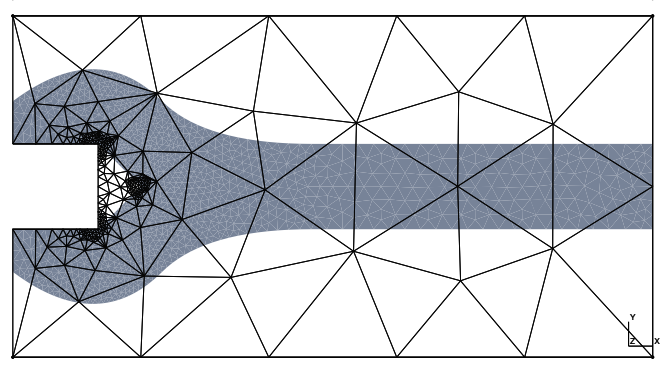

Figure 29: Boundary fitted meshes: (a) Homogeneous refinement; (b) Refined near the singular points.

(a)

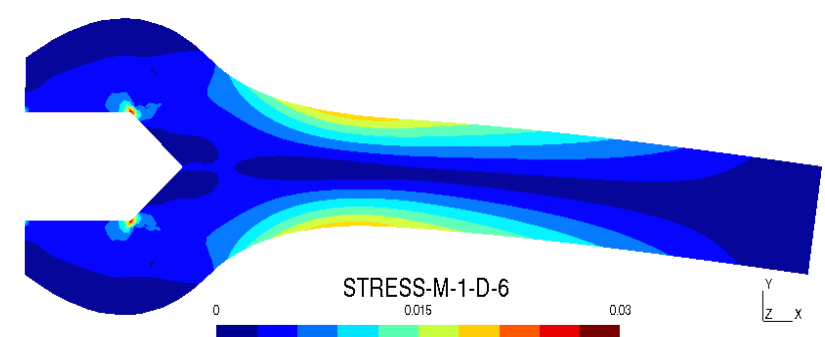

(b)

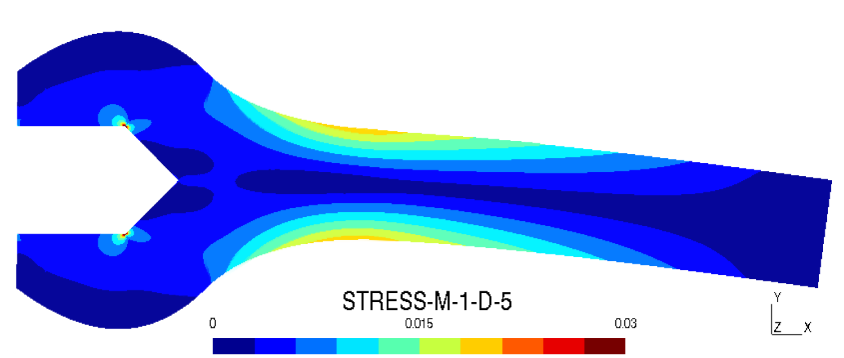

Figure 30: Boundary fitted meshes (Von-Mises norm of the stress field on the deformed configuration): (a) Homogeneous refinement; (b) Refined near the singular points. 


\subsection{Coupling NE X-FEM and Sub-Grid level sets}

As a last example, consider the coupling of the NURBS-Enhanced X-FEM and the SubGrid Level-Set approach. As presented earlier, this mixed strategy allows to consider features that are best defined in the physical space, for example evolving ones such as cracks or digitalized details coming from non-destructive image acquisitions (in the case of health monitoring). To illustrate this aspect, consider the spanner from the last example. First, three holes are introduced in the geometrically exact model, as illustrated in Figure 31(a). Sub-Grid level sets [17, 32] allows to represent with an arbitrary smoothness level these details, despite of the coarse computational mesh (see Figure 31(b)). The material properties and boundary conditions are assumed to be the same as in the last example. A $p=6$ polynomial order approximation is considered, which leads to an amount of 9301 dofs. The Von-Mises norm of the stress field on the deformed configuration is depicted in Figure 32(b). A reference quadratic FEM computation is obtained using an extremely fine mesh (1540591 dofs), and a second one is also conducted on the mesh depicted in Figure 31(c) (11 259 dofs). The solutions of these computations are represented in Figure 32(a-b). It can be seen that there is a very good agreement between them. The discrepancy in the strain energy between the reference computation and the NE X-FEM one is $0.57543 \%$, and the error for the quadratic solution is 0.66547 . As shown in the last section, the proper enforcement of the boundary conditions near the head of the spanner and the use of a properly refined mesh would improve the results.

Finally, a crack is inserted in the spanner in order to illustrate the ability of the approach to handle both exact geometrical description and features that can evolve in the physical space (the location of this crack is presented in Figure 33). Topological enrichment is considered [4], and the four classical near-tip enrichment functions proposed in [36] are used on top of a $P 5$ approximation. The material properties and boundary conditions are kept as in the previous example. The solution is then depicted in Figures 34. It can be seen that the singularities near the crack tips are nicely captured because of the enrichment and despite of the coarse computational mesh. 
(a)

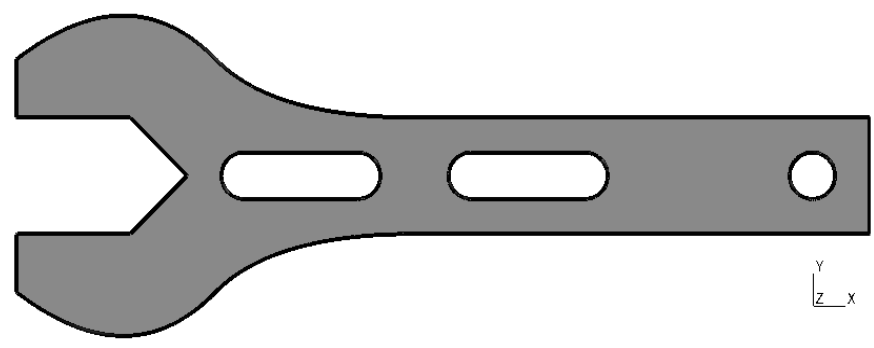

(b)

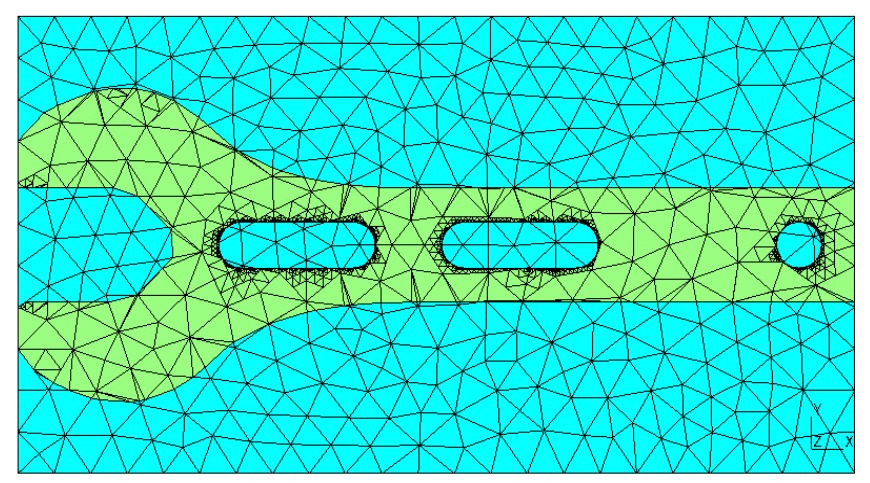

(c)

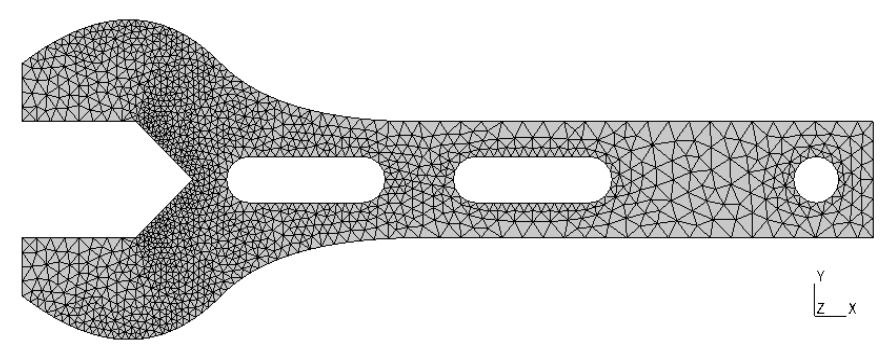

Figure 31: Spanner and voids: (a) Geometry; (b) Computational mesh and integration cells (NURBS-Enhanced for the outer boundary, and Sub-Grid Level-Set for the holes); (c) Mesh used for the quadratic computation. 
(a)

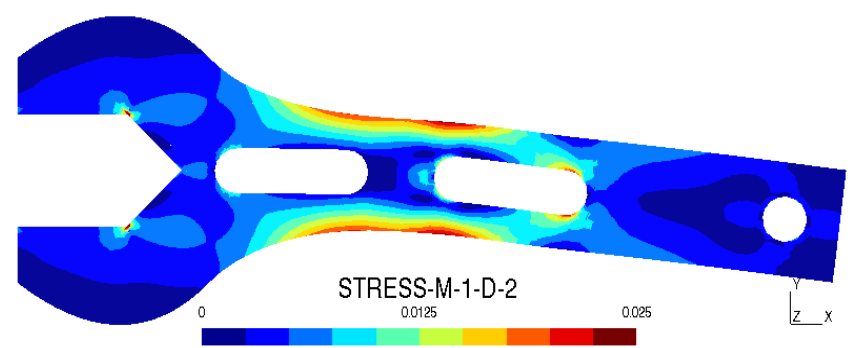

(b)

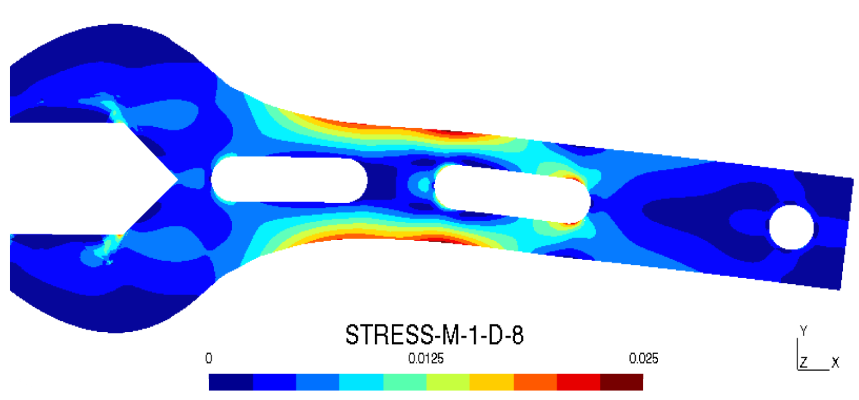

Figure 32: Spanner and voids: (a) Quadratic FEM solution (b) X-FEM solution. (VonMises norm of the stress field on the deformed configuration)

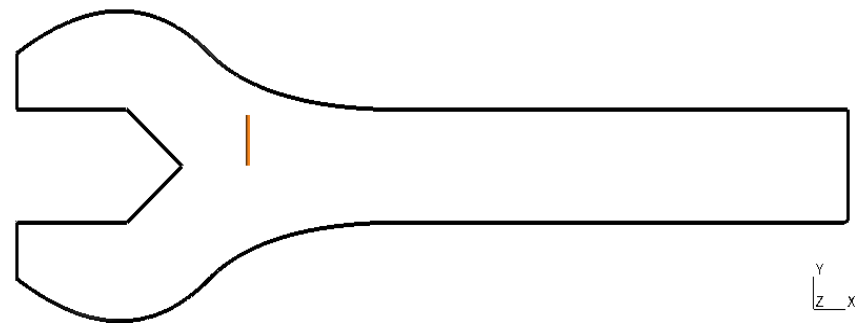

Figure 33: Cracked spanner: location of the crack. 
(a)

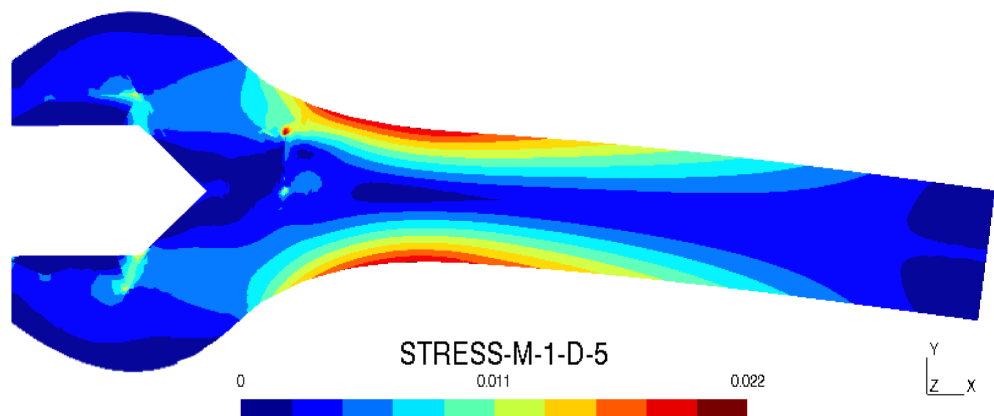

(b)

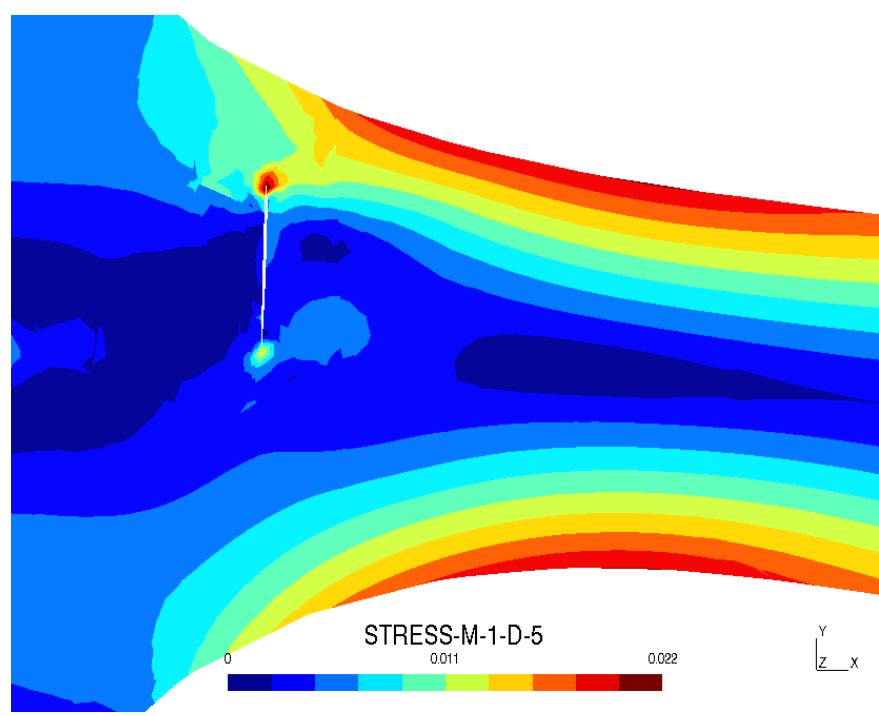

(c)

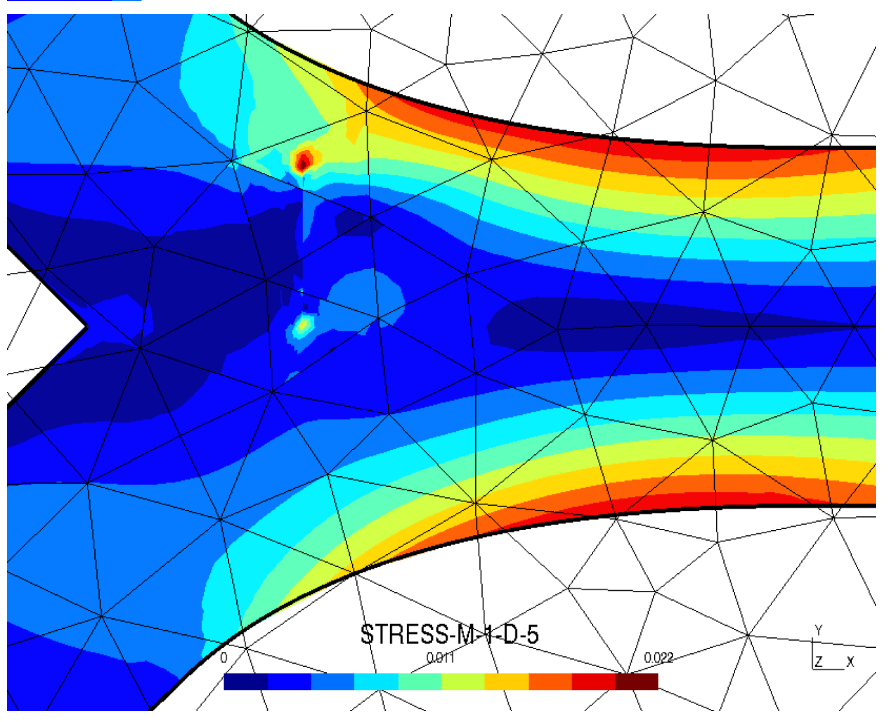

Figure 34: Cracked spanner (Von-Mises norm of the stress field): (a) X-FEM solution; (b) Zoom on the cracked region; (c) Crack and mesh on the undeformed configuration. 


\section{CONCLUSION}

An eXtended Finite Element approach was proposed for 2D unfitted geometrically exact analysis. This approach relies on two main ingredients: (i) the use of the exact boundaries by means of NURBS and Nurbs-Enhanced subelements and (ii) the use of a partition of unity approach for enriching the non-conforming solution. With respect to isogeometric analysis, the proposed approach simplifies greatly the mesh refinement process. In addition, only the surface parametrization of the domain is necessary, which is the only information a CAD modeller is working on. Finally, only a small part of the $\mathrm{X}$-FEM code has to be modified. The NURBS-enhanced mapping is also more versatile than the blending function mapping proposed in [54] in the case of breakpoints or trimmed NURBS inside an element (see $[51,50]$ ), especially in 3D. It was demonstrated that the use of a straight sided background mesh allows to define a consistent high-order approximation without the need of cartesian-based shape functions. Optimal convergence rates could be obtained. In addition, the knowledge of the parametrization of the surface allows to predict automatically the location of singular points in the solution, and enrich the approximation accordingly. It was also proposed to use an implicit/explicit representation of the geometry: explicit for the general geometry of the structure and implicit for evolving features such as cracks. Great flexibility is added thanks to this mixed strategy. Work will now focus on the extension to the 3D case: the extension of the NURBS-enhanced mapping was already demonstrated in [50], so that this extension would depend on the ease of implementation in this more complex context. In the case where the regularity of the solution is of interest, arbitrary smooth approximation can also be considered if a structured mesh and Spline-based shape functions are used. In this case, it is not necessary to extend the NURBS-enhanced mapping to quadrangles and hexaedra as proposed in [49]: The quadrangles (resp. hexaedra) can be split into two triangles (resp. six tetraedra) for the integration before applying the proposed approach on this simplex mesh. Finally, note that the approach can be directly applied in 3D for thin-walled structures, following the approach proposed by Rank et al. [42] with the Finite Cell method.

Acknowledgement:

The support of the ERC Advanced Grant XLS no 291102 is gratefully acknowledged 


\section{REFERENCES}

\section{References}

[1] Babuška I, Banerjee U (2012) Stable Generalized Finite Element Method (SGFEM). Computer Methods in Applied Mechanics and Engineering 201-204:91-111, DOI 10.1016/j.cma.2011.09.012, URL http://dx.doi.org/10.1016/j.cma.2011.09.012

[2] Bazilevs Y, Bajaj C, Calo V, Hughes T (2010) Special issue on computational geometry and analysis. Computer Methods in Applied Mechanics and Engineering 199(5-8):223-, DOI 10.1016/j.cma.2009.10.006, URL http://www.sciencedirect.com/science/article/pii/S0045782509003429

[3] Bazilevs Y, Calo V, Cottrell J, Evans J, Hughes T, Lipton S, Scott M, Sederberg $\mathrm{T}$ (2010) Isogeometric analysis using T-splines. Computer Methods in Applied Mechanics and Engineering 199(5-8):229-263, DOI 10.1016/j.cma.2009.02.036, URL http://www.sciencedirect.com/science/article/pii/S0045782509000875

[4] Béchet E, Minnebo H, Moës N, Burgardt B (2005) Improved implementation and robustness study of the X-FEM for stress analysis around cracks. International Journal for Numerical Methods in Engineering 64(8):1033-1056

[5] Béchet E, Moës N, Wohlmuth B (2009) A stable lagrange multiplier space for the stiff interface conditions within the extended finite element method. International Journal for Numerical Methods in Engineering 78(8):931-954, DOI $10.1002 /$ nme. 2515

[6] Belytschko T, Parimi C, Moës N, Usui S, Sukumar N (2003) Structured extended finite element methods of solids defined by implicit surfaces. International Journal for Numerical Methods in Engineering 56:609-635

[7] Benson DJ, Bazilevs Y, De Luycker E, Hsu MC, Scott M, Hughes TJR, Belytschko T (2010) A generalized finite element formulation for arbitrary basis functions: From isogeometric analysis to XFEM. International Journal for Numerical Methods in Engineering 83(6):765-785, DOI 10.1002/nme.2864, URL http://dx.doi.org/10.1002/nme.2864

[8] Boor CD (1972) On calculation with $B$-splines. Journal of Approximation Theory 6:50-62

[9] Cheng KW, Fries T (2009) Higher-order XFEM for curved strong and weak discontinuities. International Journal for Numerical Methods in Engineering 82:564-590, DOI 10.1002/nme.2768, URL http://doi.wiley.com/10.1002/nme.2768

[10] Chessa J, Wang H, Belytschko T (2003) On the construction of blending elements for local partition of unity enriched finite elements. International Journal for Numerical Methods in Engineering 57:1015-1038 
[11] Ciarlet P, Raviart PA (1972) Interpolation theory over curved elements, with applications to finite element methods. Computer Methods in Applied Mechanics and Engineering 1(2):217-249, DOI 10.1016/0045-7825(72)90006-0, URL http://www.sciencedirect.com/science/article/pii/0045782572900060

[12] Cottrell J, Hughes TJ, Bazilevs Y (2009) Isogeometric Analysis: Toward Integration of CAD and FE. John Wiley \& Sons

[13] Cowper G (1973) Gaussian Quadrature Formulas for Triangles. International Journal for Numerical Methods in Engineering 7:405-408

[14] Cox M (1971) The numerical evaluation of B-splines. Tech. Rep. DNAC 4, National Physical Laboratory

[15] De Luycker E, Benson DJ, Belytschko T, Bazilevs Y, Hsu MC (2011) X-FEM in isogeometric analysis for linear fracture mechanics. International Journal for Numerical Methods in Engineering 87(6):541-565, DOI 10.1002/nme.3121, URL http://dx.doi.org/10.1002/nme.3121

[16] Dolbow J, Harari I (2009) An efficient finite element method for embedded interface problems. International Journal for Numerical Methods in Engineering 78(2):229 252, DOI 10.1002/nme.2486, URL http://dx.doi.org/10.1002/nme.2486

[17] Dréau K, Chevaugeon N, Moës N (2010) Studied X-FEM enrichment to handle material interfaces with higher order finite element. Computer Methods in Applied Mechanics and Engineering 199(29-32):1922-1936, DOI 10.1016/j.cma.2010.01.021, URL http://linkinghub.elsevier.com/retrieve/pii/S0045782510000563

[18] Duster A, Parvizian J, Yang Z, Rank E (2008) The finite cell method for threedimensional problems of solid mechanics. Computer Methods in Applied Mechanics and Engineering 197(45-48):3768-3782, DOI 10.1016/j.cma.2008.02.036, URL http://linkinghub.elsevier.com/retrieve/pii/S0045782508001163

[19] Ergatoudis I, Irons B, Zienkiewicz O (1968) Curved, isoparametric, "quadrilateral" elements for finite element analysis. International Journal of Solids and Structures 4(1):31-42, DOI 10.1016/0020-7683(68)90031-0, URL http://www.sciencedirect.com/science/article/pii/0020768368900310

[20] Forsey DR, Bartels RH (1988) Hierarchical B-spline refinement. SIGGRAPH Comput Graph 22(4):205-212, DOI 10.1145/378456.378512, URL http://doi.acm.org.gate6.inist.fr/10.1145/378456.378512

[21] Fries T (2008) A corrected XFEM approximation without problems in blending elements. International Journal for Numerical Methods in Engineering 75(5):503532, DOI 10.1002/nme.2259, URL http://dx.doi.org/10.1002/nme.2259 
[22] Fries TP, Belytschko T (2010) The extended/generalized finite element method: An overview of the method and its applications. International Journal for $\mathrm{Nu}-$ merical Methods in Engineering 84(3):253-304, DOI 10.1002/nme.2914, URL http://dx.doi.org/10.1002/nme.2914

[23] Ghorashi SS, Valizadeh N, Mohammadi S (2012) Extended isogeometric analysis for simulation of stationary and propagating cracks. International Journal for Numerical Methods in Engineering 89(9):1069-1101, DOI 10.1002/nme.3277, URL http://dx.doi.org/10.1002/nme.3277

[24] Gomez H, Calo VM, Bazilevs Y, Hughes TJ (2008) Isogeometric analysis of the Cahn-Hilliard phase-field model. Computer Methods in Applied Mechanics and Engineering 197(49-50):4333-4352, DOI 10.1016/j.cma.2008.05.003, URL http://www.sciencedirect.com/science/article/pii/S0045782508001953

[25] Haasemann G, Kästner M, Prüger S, Ulbricht V (2011) Development of a quadratic finite element formulation based on the XFEM and NURBS. International Journal for Numerical Methods in Engineering 86(4-5):598-617, DOI 10.1002/nme.3120, URL http://dx.doi.org/10.1002/nme.3120

[26] Hansbo A, Hansbo P (2004) A finite element method for the simulation of strong and weak discontinuities in solid mechanics. Computer Methods in Applied Mechanics and Engineering 193(33-35):3523-3540, DOI 10.1016/j.cma.2003.12.041, URL http://www.sciencedirect.com/science/article/B6V29-4BRSGX04/2/0a5f9d036b9ef16b2a0e571b247ff04d

[27] Huerta A, Casoni E, Sala-Lardies E, Fernandez-Mendez S, Peraire J (2010) Modeling discontinuities with high-order elements. In: ECCM 2010 - Paris

[28] Hughes T, Cottrell J, Bazilevs Y (2005) Isogeometric analysis: CAD, finite elements, NURBS, exact geometry and mesh refinement. Computer Methods in Applied Mechanics and Engineering 194(39-41):4135-4195, DOI 10.1016/j.cma.2004.10.008, URL http://www.sciencedirect.com/science/article/pii/S0045782504005171

[29] Kim HJ, Seo YD, Youn SK (2009) Isogeometric analysis for trimmed CAD surfaces. Computer Methods in Applied Mechanics and Engineering 198(37-40):2982-2995, DOI 10.1016/j.cma.2009.05.004, URL http://www.sciencedirect.com/science/article/pii/S0045782509001856

[30] Kim HJ, Seo YD, Youn SK (2010) Isogeometric analysis with trimming technique for problems of arbitrary complex topology. Computer Methods in Applied Mechanics and Engineering 199(45-48):2796-2812, DOI 10.1016/j.cma.2010.04.015, URL http://www.sciencedirect.com/science/article/pii/S0045782510001325

[31] Királyfalvi G, Szabó B (1997) Quasi-regional mapping for the pversion of the finite element method. Finite Elements in Analysis and Design 27(1):85-97, DOI 10.1016/S0168-874X(97)00006-1, URL http://linkinghub.elsevier.com/retrieve/pii/S0168874X97000061 
[32] Legrain G, Chevaugeon N, Dréau K (2012) High order X-FEM and levelsets for complex microstructures: Uncoupling geometry and approximation. Computer Methods in Applied Mechanics and Engineering 241-244(0):172-189, DOI 10.1016/j.cma.2012.06.001, URL http://www.sciencedirect.com/science/article/pii/S0045782512001880

[33] Lenoir M (1986) Optimal Isoparametric Finite Elements and Error Estimates for Domains Involving Curved Boundaries. SIAM Journal on Numerical Analysis 23(3):562-580, DOI 10.1137/0723036, URL http://link.aip.org/link/?SNA/23/562/1

[34] Lipton S, Evans J, Bazilevs Y, Elguedj T, Hughes T (2010) Robustness of isogeometric structural discretizations under severe mesh distortion. Computer Methods in Applied Mechanics and Engineering 199(5-8):357-373, DOI 10.1016/j.cma.2009.01.022, URL http://www.sciencedirect.com/science/article/pii/S0045782509000346

[35] Melenk J, Babuška I (1996) The partition of unity finite element method : Basic theory and applications. Comp Meth in Applied Mech and Engrg 139:289-314

[36] Moës N, Dolbow J, Belytschko T (1999) A finite element method for crack growth without remeshing. International Journal for Numerical Methods in Engineering $46: 131-150$

[37] Moës N, Cloirec M, Cartraud P, Remacle JF (2003) A computational approach to handle complex microstructure geometries. Comp Meth in Applied Mech and Engrg 192:3163-3177, URL http://dx.doi.org/doi:10.1016/S0045-7825(03)00346-3

[38] Moës N, Béchet E, Tourbier M (2006) Imposing Dirichlet boundary conditions in the extended finite element method. International Journal for Numerical Methods in Engineering 67(12):1641-1669

[39] Moumnassi M, Belouettar S, Béchet É, Bordas SP, Quoirin D, Potier-Ferry M (2011) Finite element analysis on implicitly defined domains: An accurate representation based on arbitrary parametric surfaces. Computer Methods in Applied Mechanics and Engineering 200(5-8):774-796, DOI 10.1016/j.cma.2010.10.002, URL http://www.sciencedirect.com/science/article/pii/S004578251000280X

[40] Nitsche J (1971) Über ein Variationprinzip zur lösung von Dirichlet-Problem bei Verwendung von Teilräumen, die keinen Randbedingungen unterworfen sind. Abh Math Sem Univ Hamburg 36:9-15

[41] Parvizian J, Duster A, Rank E (2007) Finite cell method - h and $\mathrm{p}$ extension for embedded domain problems in solid mechanics. Computational Mechanics 41(1):121-133, DOI 10.1007/s00466-007-0173-y, URL http://www.springerlink.com/index/10.1007/s00466-007-0173-y 
[42] Rank E, Ruess M, Kollmannsberger S, Schillinger D, Düster A (2012) Geometric modeling, isogeometric analysis and the finite cell method. Computer Methods in Applied Mechanics and Engineering 249-252:104-115, DOI 10.1016/j.cma.2012.05.022, URL http://linkinghub.elsevier.com/retrieve/pii/S0045782512001855

[43] Sala-Lardies E, Huerta A (2012) Optimally Convergent High-Order X-FEM for Problems with Voids and Inclusions. In: Eccomas 2012

[44] Schillinger D, Rank E (2011) An unfitted hp-adaptive finite element method based on hierarchical B-splines for interface problems of complex geometry. Computer Methods in Applied Mechanics and Engineering 200(47-48):3358-3380, DOI 10.1016/j.cma.2011.08.002, URL http://www.sciencedirect.com/science/article/pii/S004578251100257X

[45] Schillinger D, Düster A, Rank E (2011) The hp-d-adaptive finite cell method for geometrically nonlinear problems of solid mechanics. International Journal for Numerical Methods in Engineering pp n/a-n/a, DOI 10.1002/nme.3289, URL http://dx.doi.org/10.1002/nme.3289

[46] Schillinger D, Dedè L, Scott MA, Evans JA, Borden MJ, Rank E, Hughes TJ (2012) An isogeometric design-through-analysis methodology based on adaptive hierarchical refinement of NURBS, immersed boundary methods, and T-spline CAD surfaces. Computer Methods in Applied Mechanics and Engineering (0):-, DOI 10.1016/j.cma.2012.03.017, URL http://www.sciencedirect.com/science/article/pii/S004578251200093X

[47] Sederberg TW, Zheng J, Bakenov A, Nasri A (2003) T-splines and TNURCCs. ACM Trans Graph 22(3):477-484, DOI 10.1145/882262.882295, URL http://doi.acm.org.gate6.inist.fr/10.1145/882262.882295

[48] Sevilla R, Fernández-Méndez S (2011) Numerical integration over 2D NURBSshaped domains with applications to NURBS-enhanced FEM. Finite Elements in Analysis and Design 47(10):1209-1220, DOI 10.1016/j.finel.2011.05.011, URL http://www.sciencedirect.com/science/article/pii/S0168874X1100117X

[49] Sevilla R, Fernández-Méndez S, Huerta A (2008) NURBS-enhanced finite element method (NEFEM). International Journal for Numerical Methods in Engineering 76(1):56-83, DOI 10.1002/nme.2311, URL http://dx.doi.org/10.1002/nme.2311

[50] Sevilla R, Fernández-Méndez S, Huerta A (2011) 3D NURBS-enhanced finite element method (NEFEM). International Journal for Numerical Methods in Engineering 88(2):103-125, DOI 10.1002/nme.3164, URL http://dx.doi.org/10.1002/nme.3164

[51] Sevilla R, Fernández-Méndez S, Huerta A (2011) Comparison of high-order curved finite elements. International Journal for Numerical Methods in Engineering 87(8):719-734, DOI 10.1002/nme.3129, URL http://dx.doi.org/10.1002/nme.3129 
[52] Seweryn A, Molski K (1996) Elastic stress singularities and corresponding generalized stress intensity factors for angular corners under various boundary conditions. Engineering $\{\mathrm{F}\}$ racture $\{\mathrm{M}\}$ echanics 55(4):529-556, DOI 10.1016/S00137944(96)00035-5, URL http://dx.doi.org/10.1016/S0013-7944(96)00035-5

[53] Strouboulis T, Babuška I, Copps K (2000) The design and analysis of the generalized finite element method. Computer Methods in Applied Mechanics and Engineering 181:43-69

[54] Strouboulis T, Copps K, Babuška I (2001) The generalized finite element method. Computer Methods in Applied Mechanics and Engineering 190(32-33):4081-4193, DOI 10.1016/S0045-7825(01)00188-8, URL http://www.sciencedirect.com/science/article/pii/S0045782501001888

[55] Sukumar N, Chopp DL, Moës N, Belytschko T (2001) Modeling Holes and Inclusions by Level Sets in the Extended Finite Element Method. Comp Meth in Applied Mech and Engrg 190:6183-6200, URL http://dx.doi.org/10.1016/S0045-7825(01)00215-8

[56] Szabó B, Babuška I (1991) Finite Element Analysis. John Wiley \& Sons, New York

[57] Szabó B, Düster A, Rank E (2004) Encyclopedia of computational mechanics, John Wiley, chap The p-version of the Finite Element Method, pp 120-140

[58] Yazid A, Abdelkader N, Abdelmadjid H (2009) A state-of-the-art review of the X-FEM for computational fracture mechanics. Applied Mathematical Modelling 33(12):4269-4282, DOI 10.1016/j.apm.2009.02.010, URL http://www.sciencedirect.com/science/article/pii/S0307904X09000560

[59] Zienkiewicz OC, Taylor R (1991) The Finite Element Method, Vol. 1, 2, 3. McGrawHill, London 